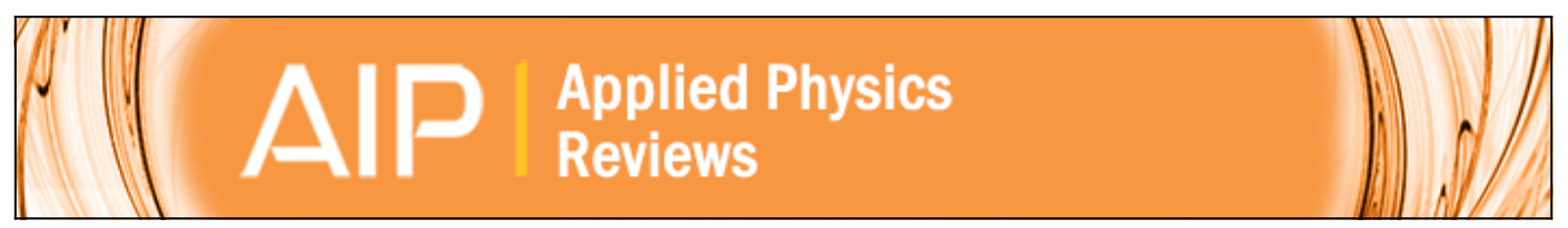

\title{
Silicene: Recent theoretical advances
}

L. C. Lew Yan Voon, Jiajie Zhu, and Udo Schwingenschlögl

Citation: Applied Physics Reviews 3, 040802 (2016); doi: 10.1063/1.4944631

View online: http://dx.doi.org/10.1063/1.4944631

View Table of Contents: http://scitation.aip.org/content/aip/journal/apr2/3/4?ver=pdfcov

Published by the AIP Publishing

\section{Articles you may be interested in}

Advanced fabrication of single-crystalline silver nanopillar on $\mathrm{SiO} 2$ substrate

Appl. Phys. Lett. 108, 043102 (2016); 10.1063/1.4940439

Advanced in-situ electron-beam lithography for deterministic nanophotonic device processing

Rev. Sci. Instrum. 86, 073903 (2015); 10.1063/1.4926995

Advances in silicon carbide science and technology at the micro- andnanoscales

J. Vac. Sci. Technol. A 31, 050805 (2013); 10.1116/1.4807902

Black thin film silicon

J. Appl. Phys. 110, 043537 (2011); 10.1063/1.3626900

Graphene-like silicon nanoribbons on $\mathrm{Ag}(110)$ : A possible formation of silicene

Appl. Phys. Lett. 96, 183102 (2010); 10.1063/1.3419932

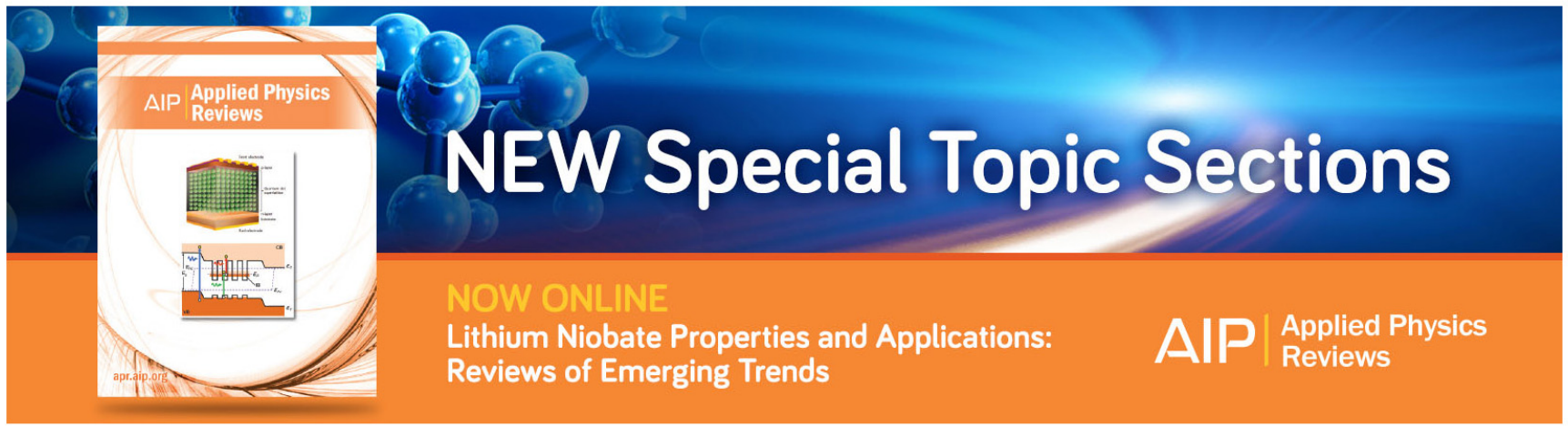




\title{
APPLIED PHYSICS REVIEWS
}

\section{Silicene: Recent theoretical advances}

\author{
L. C. Lew Yan Voon, ${ }^{1, a)}$ Jiajie Zhu, ${ }^{2}$ and Udo Schwingenschlögl ${ }^{2}$ \\ ${ }^{1}$ School of Science and Mathematics, The Citadel, 171 Moultrie St., Charleston, South Carolina 29409, USA \\ ${ }^{2}$ King Abdullah University of Science and Technology (KAUST), Physical Science and Engineering Division \\ (PSE), Thuwal 23955-6900, Saudi Arabia
}

(Received 1 December 2015; accepted 22 December 2015; published online 14 April 2016)

Silicene is a two-dimensional allotrope of silicon with a puckered hexagonal structure closely related to the structure of graphene and that has been predicted to be stable. To date, it has been successfully grown in solution (functionalized) and on substrates. The goal of this review is to provide a summary of recent theoretical advances in the properties of both free-standing silicene as well as in interaction with molecules and substrates, and of proposed device applications. (C) 2016 AIP Publishing LLC. [http://dx.doi.org/10.1063/1.4944631]

\section{TABLE OF CONTENTS}

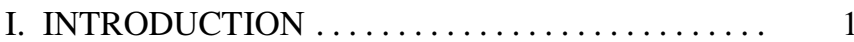

II. FREE-STANDING SILICENE. . . . . . . . . . 2

A. Structural properties .............. 2

B. Mechanical properties............... 2

C. Electronic properties............. 3

D. Phonons $\ldots \ldots \ldots \ldots \ldots \ldots \ldots \ldots \ldots \ldots$

E. Thermal properties $\ldots \ldots \ldots \ldots \ldots \ldots \ldots$

III. SILICENE NANORIBBONS ............ 5

IV. FUNCTIONALIZED SILICENE .......... 5

V. SILICENE ON SUBSTRATES ............ 8

VI. RELATIONSHIP TO SILICON AND OTHER

ALLOTROPES

VII. DEVICES . . . . . . . . . . . . . . . . . . . . 10

A. Thermoelectrics .................. 10

B. Field-effect transistors .............. 10

C. Gas adsorbent. ................. 10

D. Hydrogen storage ................ 10

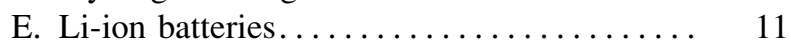

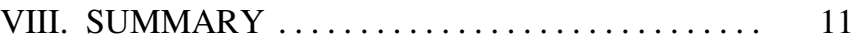

\section{INTRODUCTION}

The term "silicene" was first introduced by Guzmán-Verri and Lew Yan Voon in $2007^{1}$ to refer to a hypothetical twodimensional (2D) structure of silicon ( $\mathrm{Si}$ ) atoms in a honeycomb arrangement identical to graphene. This concept was motivated by the study of silicon nanotubes. ${ }^{2}$ A puckered structure was also investigated in Ref. 1 and referred to as Si (111), since it can be viewed as a Si (111) plane. It has been pointed out that the term "silicene" does not strictly follow the International Union of Pure and Applied Chemistry (IUPAC) nomenclature for naming organic molecules with double bonds,

\footnotetext{
a) Author to whom correspondence should be addressed. Electronic mail: llewyanv@citadel.edu
}

though one can argue that silicene is neither a molecule nor organic. Today, silicene is used broadly to denote a 2D form of silicon with a hexagonal lattice structure. An alternative naming for the same structure is a silicon monolayer sheet. ${ }^{3}$

The confusion surrounding the nomenclature reflects the unclear history of the study of silicene. The first prediction of the possible stability of a single sheet of $\mathrm{Si}$ is now widely attributed to a 1994 paper by Takeda and Shiraishi. ${ }^{4}$ In it, not only did they predict that a sheet of Si atoms will not be flat but will be puckered, or what they termed corrugated, but they also obtained the band structure of silicene (Fig. 1). Little did they know, however, that they had found the Dirac dispersion near the Fermi energy, a key property of graphene. Takeda and Shiraishi's results were repeated in 2005 by Durgun et al. ${ }^{5}$ and by Yang and $\mathrm{Ni}^{2}$ Both 2005 papers also found that even the flat (metastable) sheet would have a zero gap. The first realization that the bands are linear and, therefore, are truly Dirac-like was not until Ref. 1, when a proof was provided using both group theory and the analytical tight-

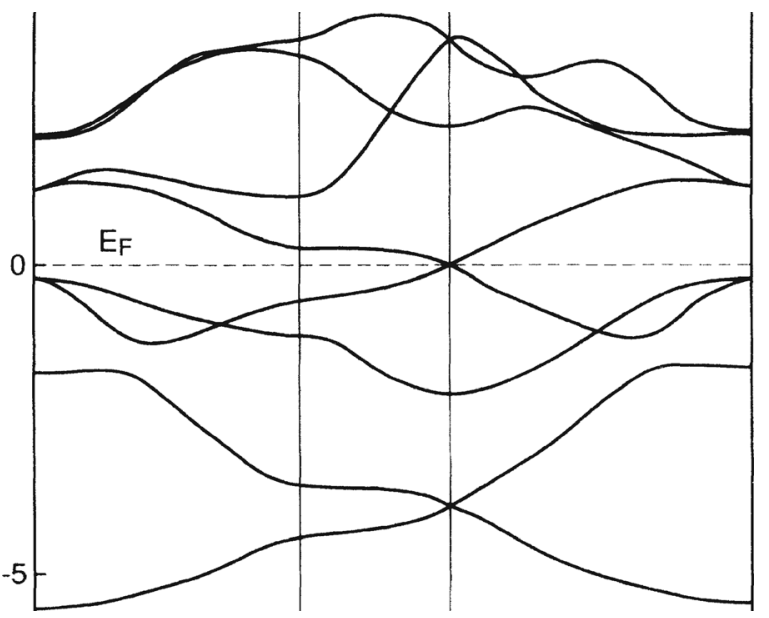

FIG. 1. Band structure of silicene. Reprinted with permission from K. Takeda and K. Shiraishi, Phys. Rev. B 50, 14916 (1994). Copyright 1994 American Physical Society. 


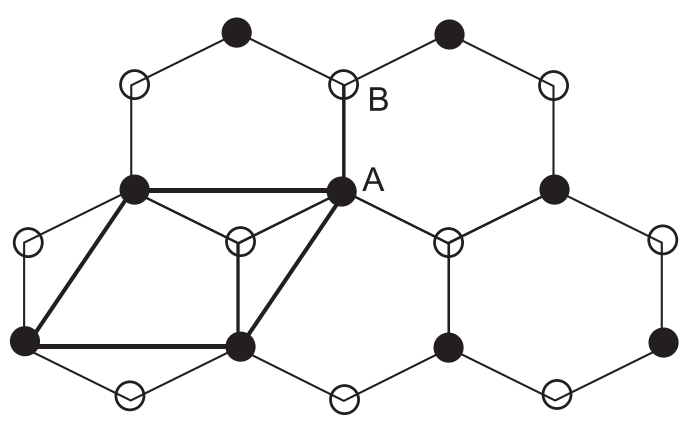

FIG. 2. Crystal structure of silicene.

binding (TB) model. The rest is history as the latter work motivated the experimental ultrahigh vacuum growth of $\mathrm{Si}$ nanoribbons on silver, ${ }^{6}$ and eventually of silicene on various metallic substrates, ${ }^{7-11}$ even though earlier experimental growth of "silicon monolayer sheets" had been reported. ${ }^{3}$

\section{FREE-STANDING SILICENE}

In this section, we provide an overview of the main physical properties of free-standing silicene.

\section{A. Structural properties}

Most calculations have predicted a hexagonal buckled structure with two atoms per unit cell as the structure of silicene (Fig. 2), with a lattice constant of $3.82 \AA$ and an out-ofplane displacement of the basis atom of $0.45 \AA$. For bulk Si, the out-of-plane $\mathrm{Si}$ atom is $0.78 \AA$ from the (111) plane. The standard predictive tool is using $a b$ initio methods for the total energy calculation and looking for minima in total energy as a function of structural parameters. The original work $^{4}$ used a $1 \times 1$ supercell, while subsequent work has also used a $2 \times 2$ supercell and molecular dynamics (MD), ${ }^{5}$ and phonon calculations. ${ }^{12}$ The $\mathrm{Si}$-Si binding energy is $4.9 \mathrm{eV} /$ atom, which is lower than that for bulk silicon (diamond structure) by $0.6 \mathrm{eV} /$ atom. $^{5}$

In 2013, Kaltsas and Tsetseris ${ }^{13}$ found that structures based on the $\sqrt{3} \times \sqrt{3}, 5 \times 5$, and $7 \times 7$ reconstructions are actually all more stable than the perfect silicene structure, by 48,17 , and $6 \mathrm{meV}$ per atom, respectively (Fig. 3).

The structure can change under the influence of external fields. For example, an in-plane biaxial strain will not only change the lattice constant but also the position of the internal atom. ${ }^{14,15}$ While an initial decrease with expansion is expected, the buckling parameter was found to increase again for a strain larger than $10 \%$ (Fig. 4). An external electrical field perpendicular to the plane also has an effect on the buckling parameter. The vertical electric field was found to increase the buckling parameter quadratically with the field. ${ }^{16}$

\section{B. Mechanical properties}

Mechanical properties of 2D materials are harder to compare to their three-dimensional (3D) forms, since there are fundamental differences in the definition of the various quantities. Thus, there are only two elastic constants instead of three for bulk Si. Two-dimensional materials are expected to have much higher mechanical strengths than bulk materials, ${ }^{15}$
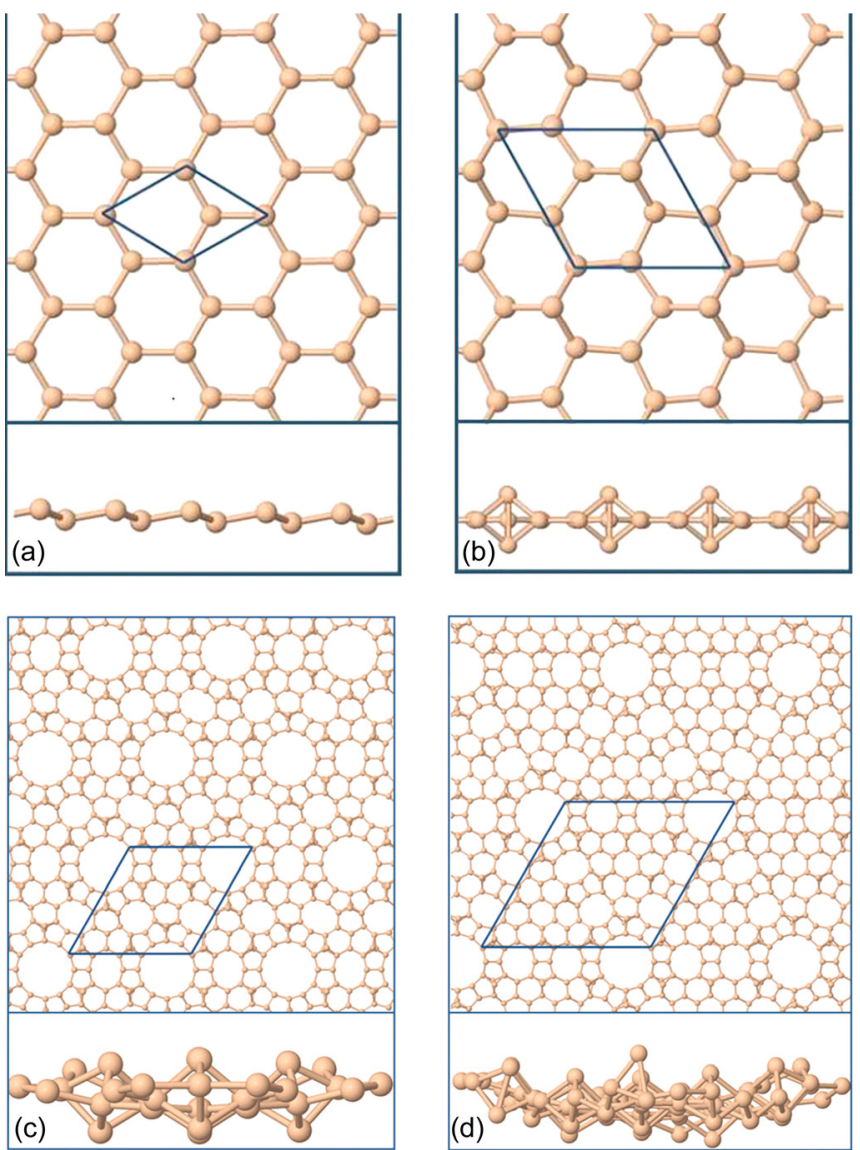

FIG. 3. Other possible stable forms of silicene: (a) silicene, (b) $\sqrt{3} \times \sqrt{3}$, (c) $5 \times 5$, and (d) $7 \times 7$. Reproduced with permission from D. Kaltsas and L. Tsetseris, Phys. Chem. Chem. Phys. 15, 9710 (2013). Copyright 2013 PCCP Owner Societies.

yet they should also have transversal flexibility. The ability to support large strain means the nonlinear elastic regime can easily be attained.

Şahin et $a l .{ }^{17}$ obtained the Poisson ratio $\nu=0.3$ and the in-plane stiffness constant $C=62 \mathrm{~J} / \mathrm{m}^{2}$. Since it is difficult to compare to $\mathrm{Si}$, we compare instead to graphene, with values of 0.16 and $335 \mathrm{~J} / \mathrm{m}^{2}$, respectively.

The anharmonic regime is reached for a biaxial strain in the range $-2 \%$ to $4 \% .^{19}$ From an analysis of the phonon

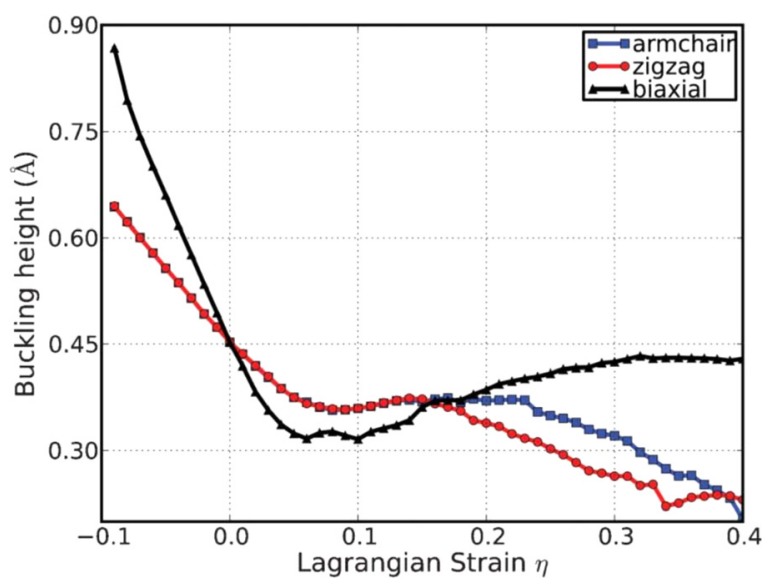

FIG. 4. Variation of buckling height with strain. Reproduced with permission from Peng et al., RSC Adv. 3, 13772 (2013). Copyright 2013 The Royal Society of Chemistry. 
frequencies, Liu et al. ${ }^{18}$ determined that silicene would be unstable under a compressive biaxial strain larger than 5\%. When the strain is larger than $17 \%,{ }^{14}$ the strain energy decreases sharply with increasing strain, and this corresponds to the yield point.

Mechanical response under a uniaxial strain has also been computed. ${ }^{15,20-23}$ The strain along perpendicular directions, conventionally taken to be the zigzag (ZZ) and armchair (AC) directions, could lead to different responses. For example, Qin $e t a l .{ }^{21}$ found that the Poisson ratio is isotropic and constant for low strain (below 2\%) but then decreases (increases) for AC (ZZ) strain; similar results were obtained by Yang et $a l^{22}$

\section{Electronic properties}

The band structure of silicon is very well-known: an indirect gap semiconductor with an indirect gap of $1.1 \mathrm{eV}$ at room temperature and with valleys approximated by quadratic dispersions. The band structure of silicene (in the absence of spinorbit interaction) has a zero gap at the Fermi energy, together with the valence and conduction bands crossing with linear dispersions, so-called Dirac cones; these occur at the $K$ and $K^{\prime}$ points in the hexagonal Brillouin zone (Fig. 5). The Dirac cones form valleys in the Brillouin zone, and the two degenerate bands at a given point originate from the $\mathrm{A}$ and $\mathrm{B}$ sublattices of the silicene structure. Due to the absence of mirror symmetry, $p_{z}$ states are coupled to $p_{x}$ and $p_{y}$ states, as well as $s$ states in forming the states near the Fermi energy. Inclusion of spin-orbit coupling would open a small gap of $1.55 \mathrm{meV} .^{24}$

Silicene is similar to graphene in being a Kane-Mele type 2D topological insulator (TI), ${ }^{25}$ but of course differs from silicon which is a band insulator (BI). A TI has a bulk energy gap but gapless edge states that allow correlated charge and spin transport. They can be distinguished from the more common BIs because the charge transport is protected from disorder (due to the correlation with spin), and mathematically, this can be represented by a different topological order or quantum number. Nevertheless, silicene is also different from graphene in that the buckled structure allows for more topological phases. For example, a vertical electric field opens a gap in silicene but not graphene and can, therefore, induce a topological phase transition. The phase diagram in the presence of an exchange field $M$ and an electric field $E_{z}$ is given in Fig. 6. Ezawa identified four

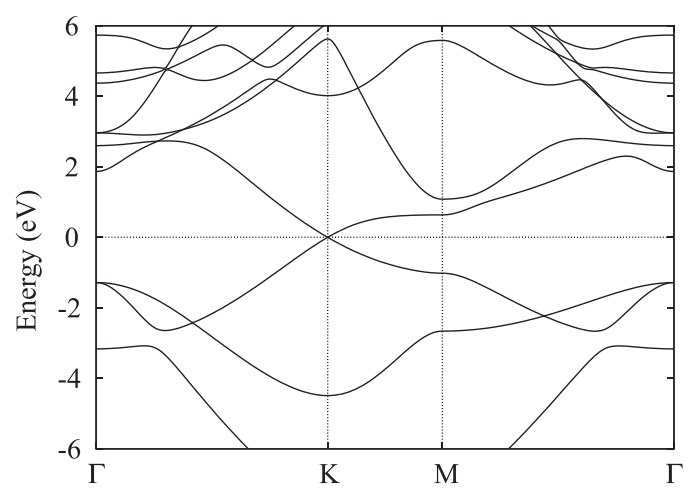

FIG. 5. Band structure of silicene.

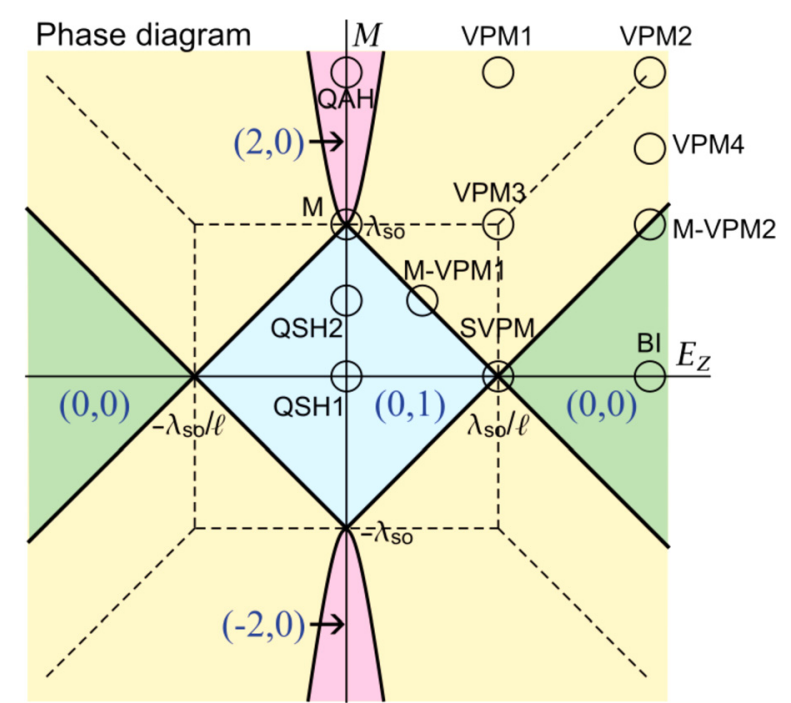

FIG. 6. Phase diagram of silicene in exchange field $(M)$ and electric field $E_{z}$. The insulator phases are indexed by the Chern and spin-Chern numbers $\left(C, C_{s}\right)$. Reprinted with permission from M. Ezawa, Phys. Rev. Lett. 109, 055502 (2012). Copyright 2012 American Physical Society.

principal phases: ${ }^{26} \mathrm{BI}$, quantum anomalous Hall (QAH), quantum spin Hall (QSH), valley-polarized metal (VPM), and spin valley-polarized metal (SVPM). The QAH is characterized by an insulating bulk gap and chiral gapless edges; it is induced by the internal magnetization and by the spin-orbit coupling, i.e., it displays quantized Hall conductance in the absence of an external magnetic field. The valley-polarized metal refers to silicene with electrons moved from the conduction band at $K$ to the valence band at $K^{\prime}$ in a perpendicular electric field.

The characterization of silicon under an external strain is also very well-known due to its importance in silicon device applications. Hydrostatic pressure changes the band gap, and uniaxial or biaxial strain can reduce the degeneracy of both the valence and conduction bands in addition to shifting them. The 2D nature of silicene has led to studies under both inplane biaxial and uniaxial strain. A biaxial tensile strain was found to lead to a semimetal-metal transition when the strain is larger than $7 \% .^{14,18,19,27}$ This is due to the lowering of the conduction band at the $\Gamma$-point; the Dirac point was found to increase in energy, ${ }^{19}$ leading to the possibility of $p$-type selfdoping. ${ }^{14}$ A biaxial compressive strain leads to a lowering of the Dirac point below the Fermi level, leading to $n$-type doping. ${ }^{27}$ The Fermi velocity is found to decrease slowly with strain, decreasing to $94 \%$ of the unstrained value for strain up to $7 \% .^{19}$ A biaxial strain is also found to lead to superconductivity. ${ }^{28} \mathrm{~A}$ uniaxial strain is expected to lead to a gap opening due to the symmetry lowering and this was initially reported. ${ }^{20,29}$ However, more recent density functional theory (DFT) calculations ${ }^{21,22}$ did not obtain a gap and interpreted the disagreement to insufficient number of $k$ points near the crossing in the earlier works. The absence of band-gap opening has been confirmed using symmetry-based $k \cdot p$ theory. ${ }^{30}$

The effect of an external electric field on the electronic structure is equally important for potential device applications such as electrooptic modulators and transistors. Electric field effects on silicene have been investigated. ${ }^{16,31-33}$ A vertical 


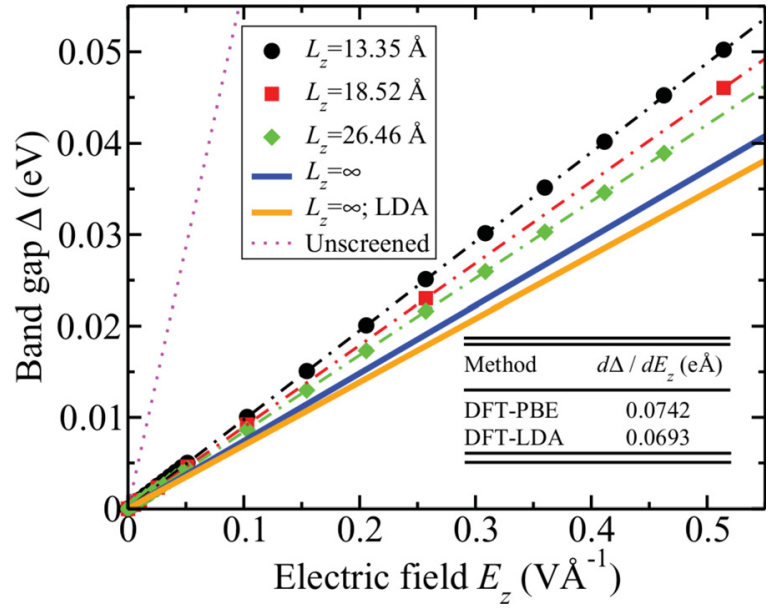

FIG. 7. Band gap as a function of external vertical electric field. Reprinted with permission from Drummond et al., Phys. Rev. B 85, 075423 (2012). Copyright 2012 American Physical Society.

electric field is expected to open a band gap in silicene, contrary to graphene, due to the fact that the two basis atoms would feel different electrostatic potentials, and the gap increased linearly with the field up to about $1 \mathrm{~V} / \AA$ (Fig. 7). The electric field also leads to an almost linear increase in the effective mass; for example, for a field of $0.4 \mathrm{~V} / \AA$, the hole mass was found to be $0.015 m_{0}\left(0.033 m_{0}\right)$ along the $K \Gamma(M K)$ direction and about $2 \%$ different for the electron mass. ${ }^{31}$

Finally, we consider the effect of an external magnetic field on the electronic properties. For free-standing silicene, the low-energy dispersion is obtained as

$$
E_{n}=\operatorname{sgn}(n) \sqrt{\frac{1}{4} \Delta_{\mathrm{SO}}^{2}+2|n| v_{F}^{2} \hbar e B}
$$

for $n \neq 0$ and $E_{n}=\sigma \eta \Delta_{\text {so }} / 2$ for $n=0$, where $n$ is the subband index (Fig. 8). The selection rules for interband transitions are found to be $\Delta n= \pm 1$.

Charge carrier transport is one of the key motivations for studying silicene given the potential for transistor action and compatibility with current Si electronics. Using a deformation potential theory and the Boltzmann transport equation, with acoustic phonons as the scattering source, an electron (a hole) mobility of $2.57 \times 10^{5} \mathrm{~cm}^{2} \mathrm{~V}^{-1} \mathrm{~s}^{-1}$ $\left(2.22 \times 10^{5} \mathrm{~cm}^{2} \mathrm{~V}^{-1} \mathrm{~s}^{-1}\right)$ at room temperature has been predicted. ${ }^{34}$ This is slightly lower than for graphene but much larger than for bulk Si.

Studies of the work function as a function of strain have been reported. ${ }^{19,21}$ The work function is defined as the minimum energy to remove an electron and is given by

$$
\Phi=E_{\mathrm{vac}}-E_{F} .
$$

The vacuum energy $E_{\text {vac }}$ was determined as the average electrostatic potential energy in a plane parallel to the silicene layer and asymptotically away. For a biaxial strain, the work function initially increased from the unstrained value of $4.8 \mathrm{eV}$, then saturates to around $5.1 \mathrm{eV}$ for a strain above $15 \% .{ }^{19}$ For a uniaxial strain, ${ }^{21}$ the change is isotropic up to $3 \%$ beyond which the $\mathrm{ZZ}$ strain leads to a larger work function but no saturation is observed.

\section{Phonons}

The dispersion relations for graphene and silicene are compared in Fig. 9. The highest G- and D-modes are $550 \mathrm{~cm}^{-1}$ and $545 \mathrm{~cm}^{-1} .{ }^{14}$ Silicene also has a flexural mode due to its $2 \mathrm{D}$ nature.

\section{E. Thermal properties}

Silicene is predicted to be stable up to $1500 \mathrm{~K}$, after which it formed a three-dimensional amorphous structure. ${ }^{35}$ Defects have been found to reduce the thermal stability of silicene by as much as $30 \% .{ }^{35}$ Passivating the defects improved the stability.

The thermal conductivity of silicene has been well studied and assumed to be mainly due to phonon transport. An inplane thermal conductivity $\kappa$ of $20 \mathrm{~W} / \mathrm{m} \mathrm{K}$ has been calculated using equilibrium MD simulation at $300 \mathrm{~K},{ }^{36}$ compared to a value of $\sim 3000-5000 \mathrm{~W} / \mathrm{m} \mathrm{K}$ for suspended graphene ${ }^{37}$ and $150-200 \mathrm{~W} / \mathrm{m} \mathrm{K}$ for silicon. The reduction has been attributed to two main causes. One is the lowering of the partial density of states (PDOS) for frequencies below $20 \mathrm{THz}$ (phonon softening), and the other is due to the blueshift in the PDOS to higher frequencies (phonon stiffening). The result is a reduction in the phonon modes present for heat conduction at low temperatures. The temperature dependence of $\kappa$ has
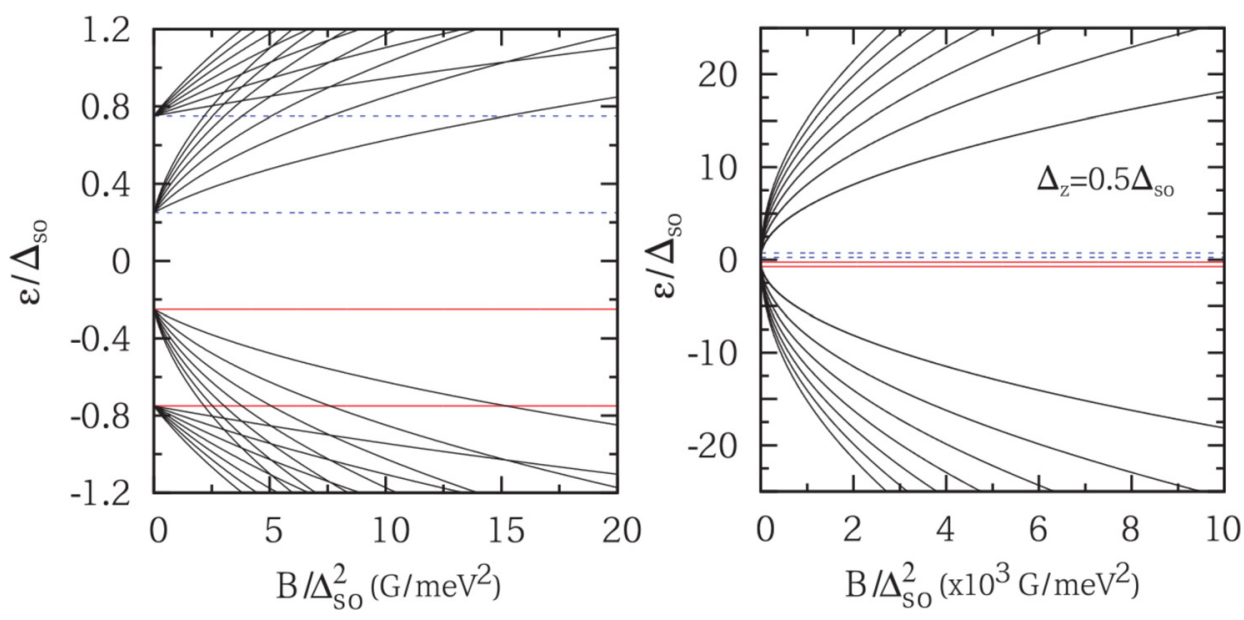

FIG. 8. Landau levels as a function of magnetic field for small (left) and large (right) field. Reprinted with permission from C. J. Tabert and E. J. Nicol, Phys. Rev. B 88, 085434 (2013). Copyright 2013 American Physical Society. ${ }^{183}$ 


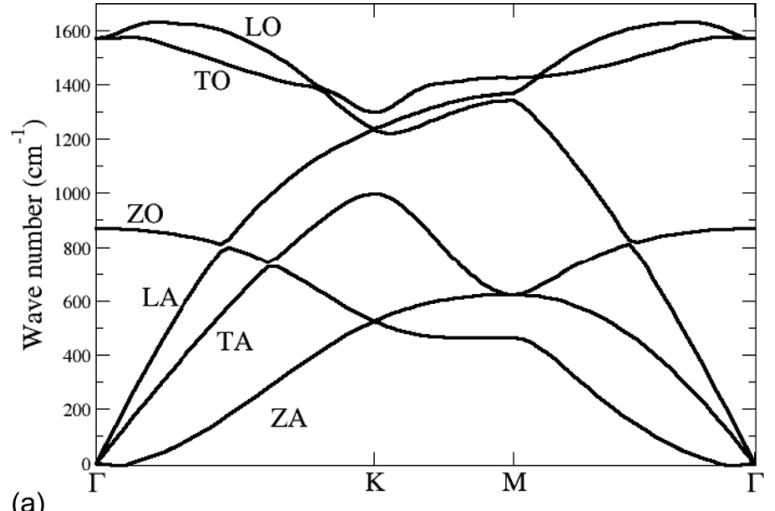

(a)

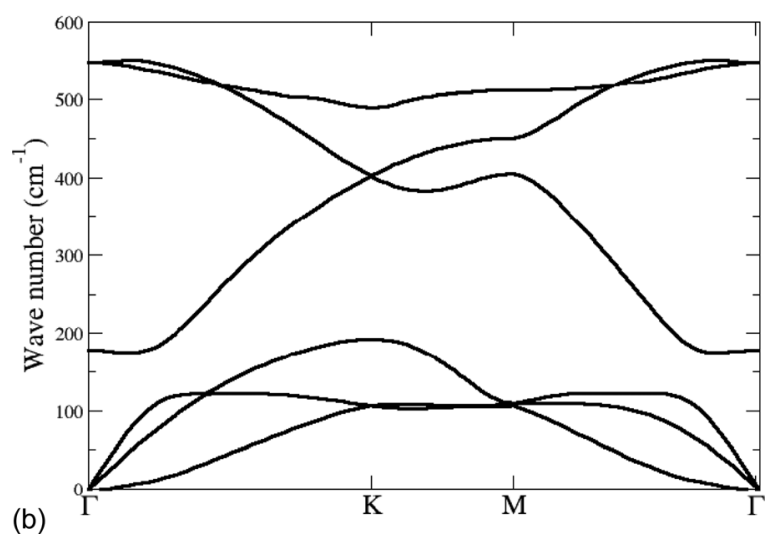

FIG. 9. Phonon spectrum for graphene (left) and silicene (right). Reprinted with permission from Gori et al., Energy Procedia 45, 512 (2014). Copyright 2014 Elsevier. ${ }^{184}$

been computed over $0<T<400 \mathrm{~K},{ }^{38}$ with a maximum value of about $100 \mathrm{~W} / \mathrm{m} \mathrm{K}$ near $100 \mathrm{~K}$, compared to around $1000 \mathrm{~W} / \mathrm{m} \mathrm{K}$ for bulk $\mathrm{Si}^{38}$ and $3000-5000 \mathrm{~W} / \mathrm{m} \mathrm{K}$ for graphene.

Thermoelectricity is measured by the figure of merit

$$
Z T=\frac{\sigma S^{2} T}{\kappa}
$$

where $\sigma$ is the electrical conductance, and $S$ is the Seebeck coefficient. The thermoelectric coefficient of silicene at room temperature has been computed ${ }^{39}$ using DFT and was found to have a $Z T_{e}$ less than 0.5 .

\section{SILICENE NANORIBBONS}

Armchair and zigzag silicene nanoribbons of width $n$ have been built by cutting along the (110) and (100) directions of silicene, respectively, as shown in Figure 10. The inplane stiffnesses are $59.1 \mathrm{~N} / \mathrm{m}$ for zigzag nanoribbon and $62.4 \mathrm{~N} / \mathrm{m}$ for armchair nanoribbon, which are much smaller than that of graphene and h-BN nanoribbons due to the $s p^{2}-s p^{3}$ hybridization. ${ }^{40,41}$ The corresponding Young's moduli for narrow nanoribbons turn out to be 140.7 and 148.5 GPa and increase with the width. Silicene nanoribbon is found to be stable under tensile strain up to $20 \%$, while a freestanding silicene nanoribbon is predicted to be subject to slight buckling ( $s p^{2}-s p^{3}$ hybridization); ${ }^{12}$ the bonding character of a supported silicene nanoribbon on $\operatorname{Ag}(110)$ substrate is still under debate. Borensztein et al. argue that the
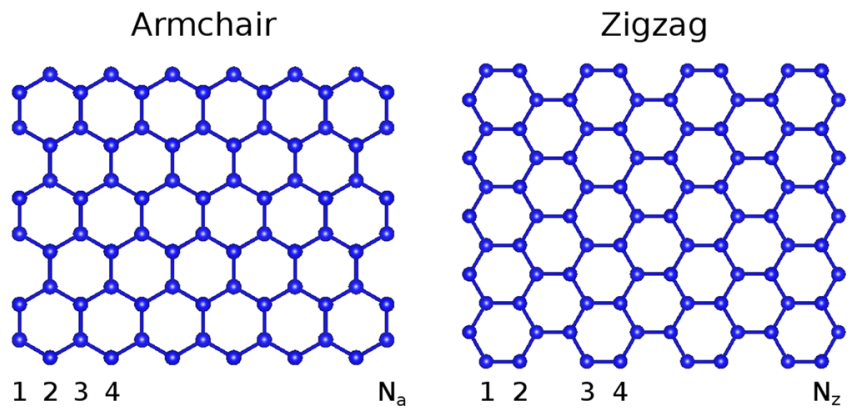

FIG. 10. Schematic illustration of armchair and zigzag silicene nanoribbons.

optical adsorption points to $s p^{3}$ hybridization, ${ }^{42}$ whereas Padova et al. favor $s p^{2}$ bonding based on results of reflection electron energy loss spectroscopy. ${ }^{43}$

An armchair silicene nanoribbon is found to be a nonmagnetic semiconductor. ${ }^{12}$ The band gap decreases with increasing width as $\Delta_{3 n+1}>\Delta_{3 n}>\Delta_{3 n+2}(n \in \mathrm{N})$, as illustrated in Figure 11, ${ }^{12,44,45}$ because of the quantum confinement and the periodicity of the Fermi wavelength. ${ }^{46}$ The band gap is predicted to be reduced by tensile strain due to decreasing charge transfer between the two sublattices. A transverse electric field can also be used to tune the band gap, where the $\pi$ electrons responsible for the top of the valence band and bottom of the conduction band accumulate at different edges of the nanoribbon. ${ }^{47}$

Different magnetic states have been predicted for a zigzag silicene nanoribbon, including ferromagnetic and antiferromagnetic states. The ground state is found to be antiferromagnetic for a narrow buckled zigzag nanoribbon, whereas the energy difference to the ferromagnetic state decreases with increasing width and a transition occurs at $N=24 .^{45,48-50}$ Flat zigzag silicene nanoribbon is ferromagnetic. ${ }^{51,53}$ In both cases, the bands near the Fermi level are due to edge states. For the antiferromagnetic state, the spin polarization decreases from the edge to the center where the two sublattices have opposite spin directions and spin-up and spin-down electrons accumulate at different edges of the nanoribbon. The band gap decreases with increasing width (Figure 11) and can be tuned by perpendicular and transversal electric fields, especially in the narrow case. ${ }^{45,48}$ The spin polarization at the edges is much larger than in the center and first increases with the width and then saturates. ${ }^{45,51,52}$

\section{FUNCTIONALIZED SILICENE}

Functionalization enables $s p^{3}$ hybridization in silicene as confirmed by the $\mathrm{Si}$ buckling height and $\mathrm{Si}-\mathrm{Si}$ bond length, ${ }^{54}$ which enhances the stability as compared to the freestanding configuration. Many adatoms have been investigated, including group I-VII elements and transition metals. Several configurations for $\mathrm{H}$-silicene (silicene fully passivated with $\mathrm{H}$ on both sides) have been investigated including top, chairlike, and boatlike configurations, see Figure 12. ${ }^{55,56}$ In the top configuration, the $\mathrm{H}$ atoms occupy one side of silicene, for the chairlike configuration, they alternate on the two sides, and for the boatlike configuration, they alternate in pairs. The chairlike and boatlike configurations are energetically almost degenerate (difference of $10 \mathrm{meV}$ per $\mathrm{Si}$ 

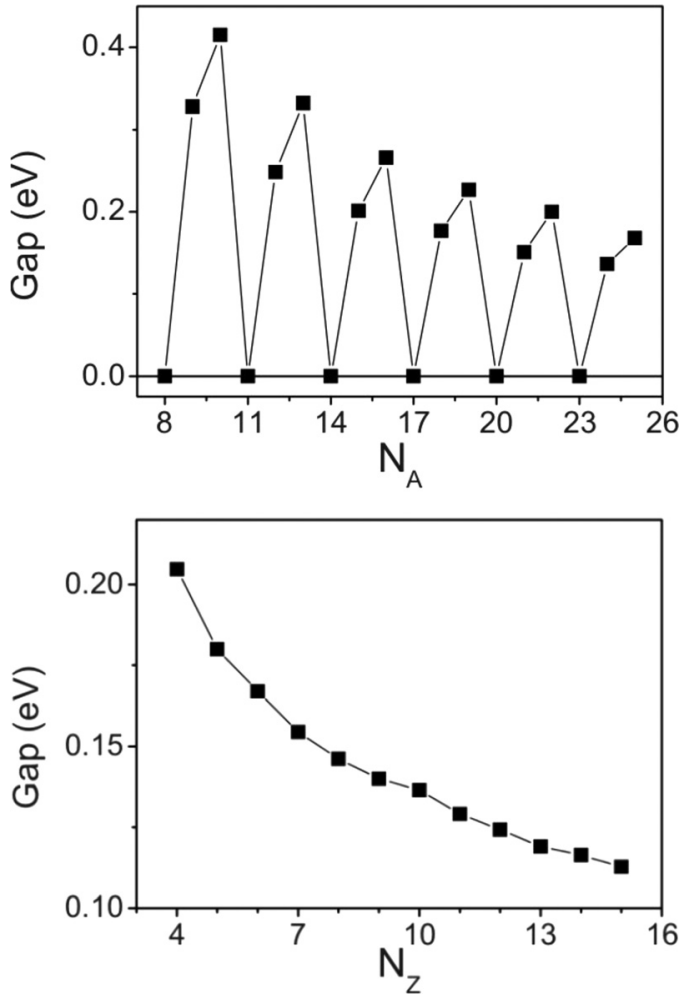

FIG. 11. Band gap of armchair (upper) and zigzag (lower) silicene nanoribbons as a function of the width. Reprinted with permission from Y. Ding and J. Ni, Appl. Phys. Lett. 95, 083115 (2009). Copyright 2009 American Institute of Physics.

atom) and much more stable than the top configuration. The chairlike configuration has an indirect gap of $2.0 \mathrm{eV}$, and the boatlike configuration has a direct gap of $1.6 \mathrm{eV}$ (Figure 13, local density approximation (LDA)). HSE and GW calculations yield two times larger band gaps, and a direct gap of $2.3 \mathrm{eV}$ has been obtained for the chairlike configuration by the generalized gradient approximation (GGA) method. ${ }^{54,57,58}$ The band gap of silicene can be tuned by means of the concentration of the $\mathrm{H}$ adatoms, ${ }^{59}$ similar to $\mathrm{H}-$ passivated bilayer silicene. ${ }^{60,61}$ The enhanced band gap (as compared to bulk $\mathrm{Si}$ ) makes $\mathrm{H}$-passivated silicene a potential candidate for electronic and optical devices.
The adatoms are stable if the binding energy is larger than the cohesive energy of the adsorbed material. Although this is the case for the $\mathrm{H}$ adatom by $0.21-0.25 \mathrm{eV}$, the energy barrier (Figure 14) for dissociation of a $\mathrm{H}_{2}$ molecule is up to $1.75 \mathrm{eV}$, so that it is difficult to have a reaction at ambient conditions. ${ }^{62,63} \mathrm{~A}$ tensile strain is predicted to reduce the energy barrier to 0.24 and $0.26 \mathrm{eV}$ under $12 \%$ biaxial strain and $13 \%$ uniaxial strain along the armchair direction, respectively. A shift of the $\mathrm{Si}$ and $\mathrm{H}$ states near the Fermi level to lower energy at the transition state (confirmed by the charge transfer) enhances the interaction and lowers the barrier. The effect is less obvious for uniaxial strain along the zigzag direction. Under tensile strain, the passivation reaction becomes more exothermic. ${ }^{62}$ In addition, a perpendicular electric field of 0.05 a.u. reduces the energy barrier to $0.38 \mathrm{eV}$ due to charge transfer from $\mathrm{Si}$ to $\mathrm{H}$, which stabilizes the $\mathrm{H}$ adatoms. ${ }^{63}$

The stability of silicene in $\mathrm{O}_{2}$ is the key for most applications. An $\mathrm{O}_{2}$ molecule is predicted to dissociate into two $\mathrm{O}$ atoms that occupy bridge sites without any energy barrier, reflecting instability of freestanding silicene in the presence of $\mathrm{O}_{2} \cdot{ }^{64}$ Since the barrier for $\mathrm{O}$ migration is $1.1 \mathrm{eV}$, desorption of $\mathrm{O}$ atom is difficult. The band gap of O-silicene is calculated to be $0.2 \mathrm{eV} .^{65,66}$ On the other hand, dissociation of an $\mathrm{O}_{2}$ molecule on $\mathrm{H}$-silicene has to overcome an energy barrier of $0.27 \mathrm{eV}$, improving the stability. ${ }^{67}$ The $\mathrm{O}$ atoms again occupy bridge sites, and migration is predicted to be impossible due to the large energy barriers introduced by the $\mathrm{H}$ atoms.

Group VII elements bind more strongly to silicene than $\mathrm{H}^{71}$ and weaken the $\mathrm{Si}$-Si interaction, leading to larger $\mathrm{Si}-\mathrm{Si}$ bonds than freestanding silicene. The band gaps of F-, $\mathrm{Cl}-$, $\mathrm{Br}$-, and I-silicene in the chairlike configuration are calculated to be $1.47,1.98,1.95$, and $1.19 \mathrm{eV}$, respectively, which are less than in $\mathrm{H}$-silicene because of the stronger bonding. The band gap turns out to grow with the concentration of $\mathrm{F}^{59}$ Asymmetric modifications of silicene with $\mathrm{H}$ and $\mathrm{F}$ atoms at different sides can realize a band gap of up to $1.82 \mathrm{eV}^{69}$

The Dirac cone is destroyed for $\mathrm{H}-, \mathrm{O}-, \mathrm{F}-, \mathrm{Cl}-, \mathrm{Br}-$, and I-silicene because of the strong interaction, ${ }^{56,65,68}$ whereas
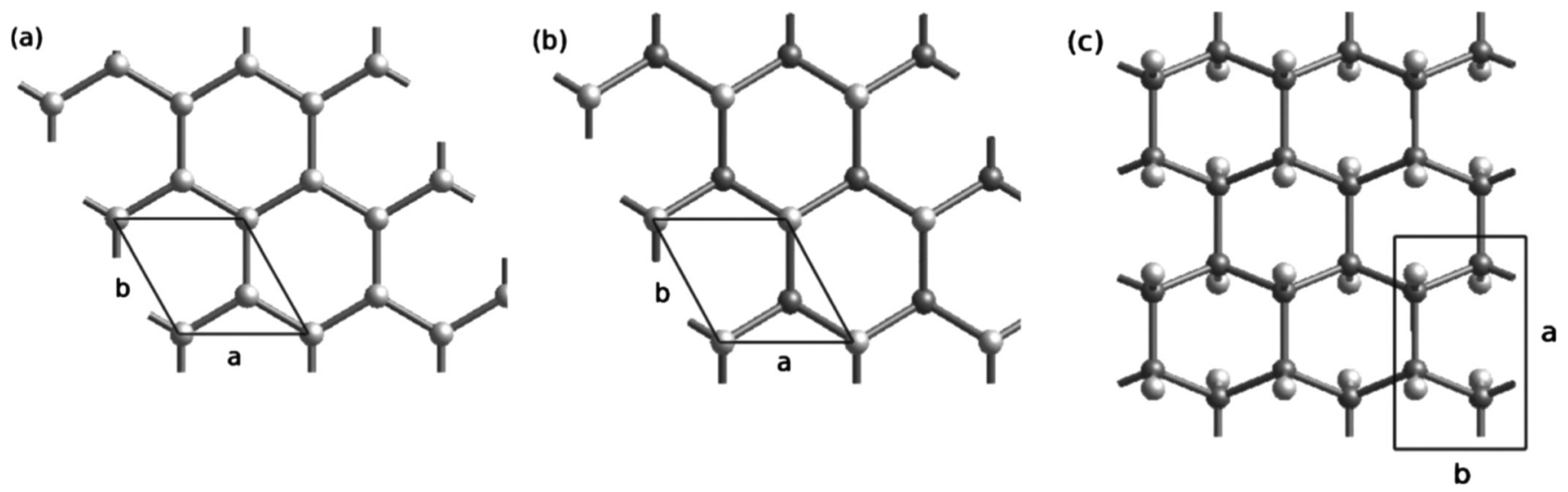

FIG. 12. H-silicene: (a) top configuration, (b) chairlike configuration, and (c) boatlike configuration, see the text for details. The Si and $\mathrm{H}$ atoms are represented by gray and white spheres, respectively. Reprinted with permission from Houssa et al., Appl. Phys. Lett. 98, 223107 (2011). Copyright 2011 American Institute of Physics. 


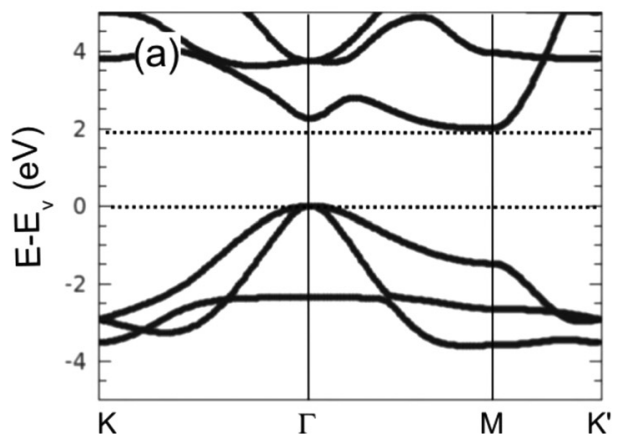

alkali metal functionalized can preserve the Dirac physics with n-type doping. ${ }^{70,71}$ The alkali metal atoms occupy hollow site ${ }^{70}$ and do not form clusters due to strong ionic interaction. $^{72,73}$ The band gap can be tuned from $0.04 \mathrm{eV}$ to $0.50 \mathrm{eV}$, where the effective mass of the charge carriers decreases for increasing band gap. ${ }^{70} \mathrm{Li}$-silicene shows no Dirac cone but a direct band gap of $0.37 \mathrm{eV}$ is opened due to the broken symmetry. ${ }^{74}$ The diffusion barrier for $\mathrm{Li}$ and $\mathrm{Na}$ migrations is calculated to be $0.25 \mathrm{eV} .^{75,76}$

Transition metal atoms are predicted to introduce spinpolarization gaps in silicene due to the localized $d$ electrons, which enables applications in spintronics. The binding energies of the elements $\mathrm{Sc}$ to $\mathrm{Zn}$ are larger than the corresponding cohesive energies, reflecting stability. ${ }^{77}$ However, Lin and $\mathrm{Ni}$ have claimed the instability of Fe adatoms. ${ }^{72} \mathrm{~A}$ halfmetallic character with band gaps of 0.51 and $0.57 \mathrm{eV}$ are obtain for $\mathrm{Fe}$ and $\mathrm{Cr}$ adsorption with low concentration. ${ }^{78}$ The corresponding magnetic moments are 2.1 and $4.0 \mu \mathrm{B}$, respectively. Moveover, it has been demonstrated that the magnetic moment introduced by $\mathrm{Cr}$ adsorption can be tuned by strain. ${ }^{79}$ Johll et al. observed a semiconducting state with a tiny band gap for Fe decoration, ${ }^{80}$ whereas $\mathrm{Ni}, \mathrm{Co}$, and $\mathrm{Mn}$
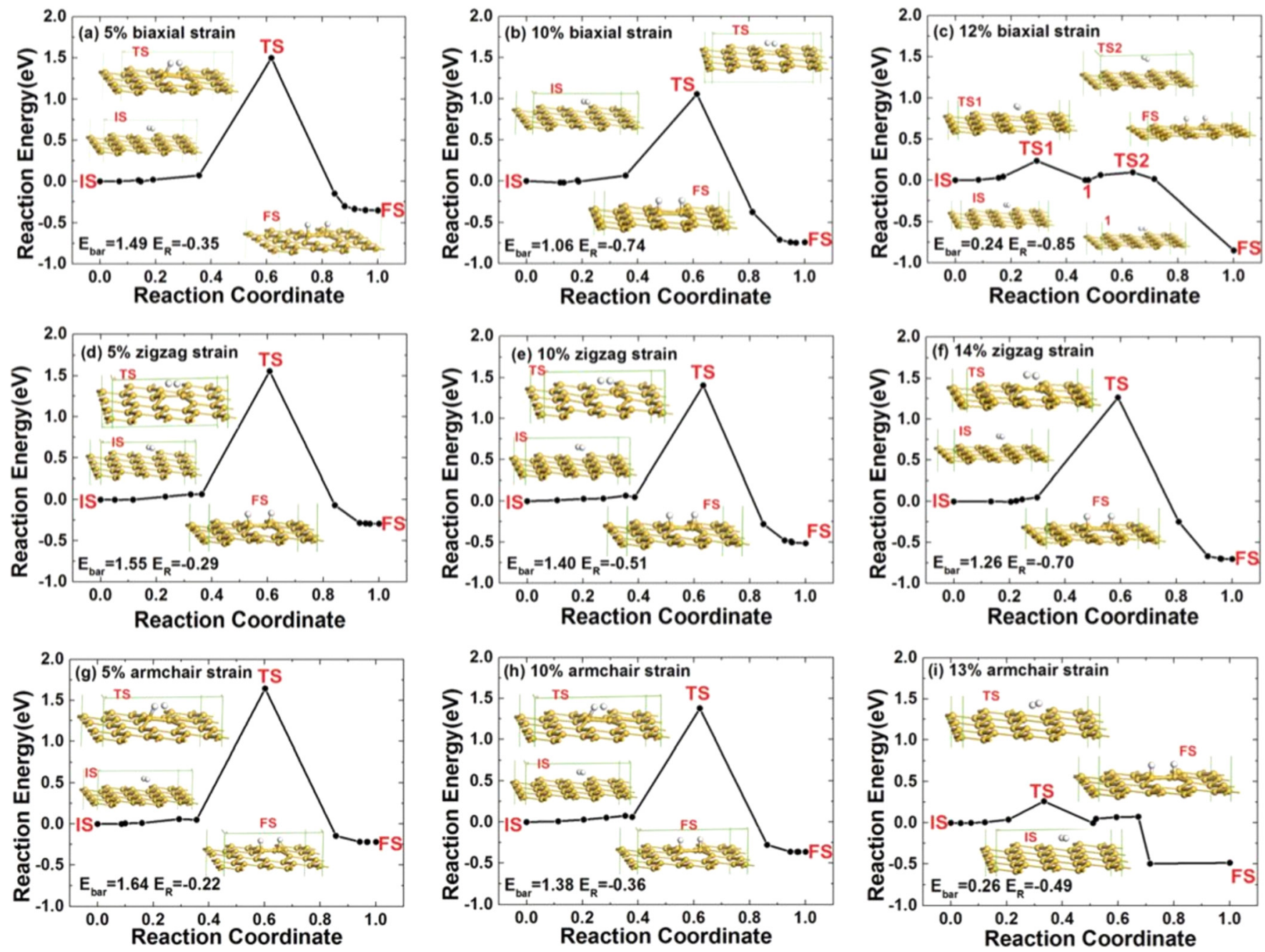

FIG. 14. Dissociative adsorption of a $\mathrm{H}_{2}$ molecule on silicene under (a)-(c) biaxial tensile strain, (d)-(f) uniaxial tensile strain along the zigzag direction, and (g)-(i) uniaxial tensile strain along the armchair direction (TS: transition state, IS: initial state, FS: final state, $\mathrm{E}_{\text {bar }}$ : energy barrier, and $\mathrm{E}_{\mathrm{R}}$ : reaction energy). The energy of the IS is set to be zero. Reproduced with permission from Wu et al., J. Mater. Chem. C 3, 2593 (2015). Copyright 2015 The Royal Society of Chemistry. 
decorated silicene are found to be metallic with magnetic moments of $0.0,1.0$, and $3.5 \mu \mathrm{B} .{ }^{80,81}$ The band gaps are opened at the Dirac point with magnetic moments ranging from 1.0 to $3.6 \mu \mathrm{B}$ for adsorption of the elements $\mathrm{Y}$ to $\mathrm{Ru},{ }^{82}$ and heavy metal adatoms lead to the band gaps to $22,10,58$, and $193 \mathrm{meV}$ for $\mathrm{Au}, \mathrm{Hg}, \mathrm{Tl}$, and $\mathrm{Pb}$, respectively. ${ }^{83}$ On the other hand, Ag is predicted to preserve a gapless Dirac cone. ${ }^{84}$ The magnetism and the band gap can also be tuned by the adsorption of organic molecules ${ }^{85-87}$ and superhalogen. ${ }^{88}$ The fact that silicene stabilizes the catalytically active planar $\mathrm{Au}_{20}$ cluster due to the strong covalent interaction can change the landscape of Au-based catalysts. ${ }^{89}$

\section{SILICENE ON SUBSTRATES}

Since $\mathrm{Si}$ atoms prefer three-dimensional structures $\left(s p^{3}\right.$ hybridization), ${ }^{90}$ freestanding silicene has not been achieved so far, and the question about appropriate substrates plays an important role. Silicene has been successfully deposited on metallic substrates, including $\operatorname{Ag}(111),{ }^{8,91-93} \operatorname{Ir}(111),{ }^{94}$ $\mathrm{ZrB}_{2}(0001),{ }^{95,96}$ and $\mathrm{ZrC}(111) .{ }^{97} \mathrm{Ag}(111)$, the most widely investigated substrate, preserves the honeycomb structure of silicene by passivating the dangling bonds of the $\mathrm{Si}$ atoms. ${ }^{90,98}$ Although overlap is found in the charge densities of the $\mathrm{Si}$ and $\mathrm{Ag}$ atoms, analysis of the electron localization function of the $\mathrm{Si}-\mathrm{Ag}$ bonds shows that the interaction is not completely covalent (partially ionic). ${ }^{99}$ This shorten the interlayer distance ( 1.23 to $1.87 \AA$ ) and can stabilize a strong buckling of silicene, amounting to more than $1 \AA$, which is much larger than the $0.44 \AA$ in the case of pristine silicene. ${ }^{100}$ Prévot et al. have argued that Ag surface atoms are released during the preparation, supporting the idea of a strong interaction between $\mathrm{Si}$ and $\mathrm{Ag}$ and therefore causing doubts on the suitability of this substrate. ${ }^{101}$ Several surface reconstructions, such as $(4 \times 4), \quad(2 \sqrt{3} \times 2 \sqrt{3}) \mathrm{R} 30^{\circ}$, $(\sqrt{13} \times \sqrt{13}) \mathrm{R} 13.9^{\circ}$, and $(\sqrt{7} \times \sqrt{7}) \mathrm{R} 19.1^{\circ}$, have been achieved by adjusting the substrate or annealing temperature. ${ }^{9,102-105}$ The stability of the reconstruction pattern is assumed to be given by the surface density, and the transformations between structures of different density have been predicted. ${ }^{106}$ A $s p^{2}-s p^{3}$ phase transition occurs before the Ag surface is fully covered by $\mathrm{Si}$ atom, which contributes to the difficulty to prepare silicene without accompanying of multilayer structures. ${ }^{107-111}$ On $\operatorname{Ir}(111)$ substrate, a $(\sqrt{7} \times \sqrt{7})$ surface reconstruction appears, and a weak interlayer interaction is predicted by the electron localization function. ${ }^{94}$ In the case of $\mathrm{ZrB}_{2}$ and $\mathrm{ZrC}$ substrates, a phase transition from $(2 \times 2)$ to $(1 \times 1)$ reconstruction is observed with decreasing temperature. The stability has been demonstrated only in terms of the phonon dispersions. ${ }^{95,97}$ The Dirac cone is lost due to strong hybridization on metallic substrates, as shown in Figure 15..$^{112-115}$ Although the $\pi$ and $\pi^{*}$ bands still can be identified for some substrates, the electronic properties are dominated by the substrates (with or without interaction). ${ }^{115,116}$ Semiconducting and insulating substrates have been explored theoretically to overcome this problem. However, there appears no Dirac cone on AlAs, AlP, GaAs, $\mathrm{GaP}, \mathrm{ZnS}, \mathrm{ZnSe}$, and AlN substrates. ${ }^{117-119}$ It is preserved on $\mathrm{GaS}$ nanosheet with a band gap of $0.17 \mathrm{eV}$, but the large lattice mismatch $(7.5 \%)$ makes the preparation difficult. ${ }^{120}$ The Dirac cone is also disturbed by a substrate band on $\mathrm{H}-$ passivated $\mathrm{Si}_{3} \mathrm{~N}_{4}(0001)$, but can be restored by n-type $\mathrm{P}$ substitution at the $\mathrm{Si}$ site with a band gap of $58 \mathrm{meV} .^{121}$ The Dirac cones are predicted on $\mathrm{H} / \mathrm{F}$-passivated $\mathrm{Si}(111)$ and $\mathrm{H}-$ passivated $\mathrm{SiC}(0001)$ substrates with lattice mismatches less than $1 \% .^{122-125} \mathrm{H}$-passivated $\mathrm{Ge}(111)$ behaves similarly, but has a larger lattice mismatch of $4 \% .{ }^{126}$ On the other hand, on Ca-passivated $\mathrm{Si}(111)$, the electrons are scattered from the Dirac point to the other points in the Brillouin zone almost without excitation energy. ${ }^{127}$ Although F-terminated $\mathrm{CaF}_{2}$ is predicted to have a Dirac cone with a band gap of $52 \mathrm{meV}$, the control of the terminal layers to saturate the dangling bonds is problematic. ${ }^{128}$

By the weak van der Waals interlayer interaction in layered materials, as compared to the strong in-plane chemical bonding, surface passivation is no longer necessary when used as a substrate for silicene, which can simplify the preparation. Graphene and $h$-BN nowadays are routinely prepared by mechanical exfoliation. ${ }^{129,130}$ Both preserve the Dirac cone of silicene with band gaps of about $50 \mathrm{meV}$ and a lattice mismatch of less than $3 \% .^{81,131-134}$ Furthermore, a Dirac cone with a band gap of $48 \mathrm{meV}$ is predicted for silicene on $\mathrm{Sc}_{2} \mathrm{CF}_{2}$ substrate (lattice mismatch of $0.92 \%$ ). ${ }^{135} \mathrm{~A}$ Dirac cone with a tiny band gap and a Fermi velocity close to that of freestanding silicene are achieved on $\mathrm{MgX}_{2}(\mathrm{X}=\mathrm{Cl}, \mathrm{Br}$, and I). ${ }^{136}$ By $\mathrm{Li}$ and $\mathrm{Na}$ doping, the band gap can be enhanced to $0.6 \mathrm{eV}$, and a binding energy comparable with
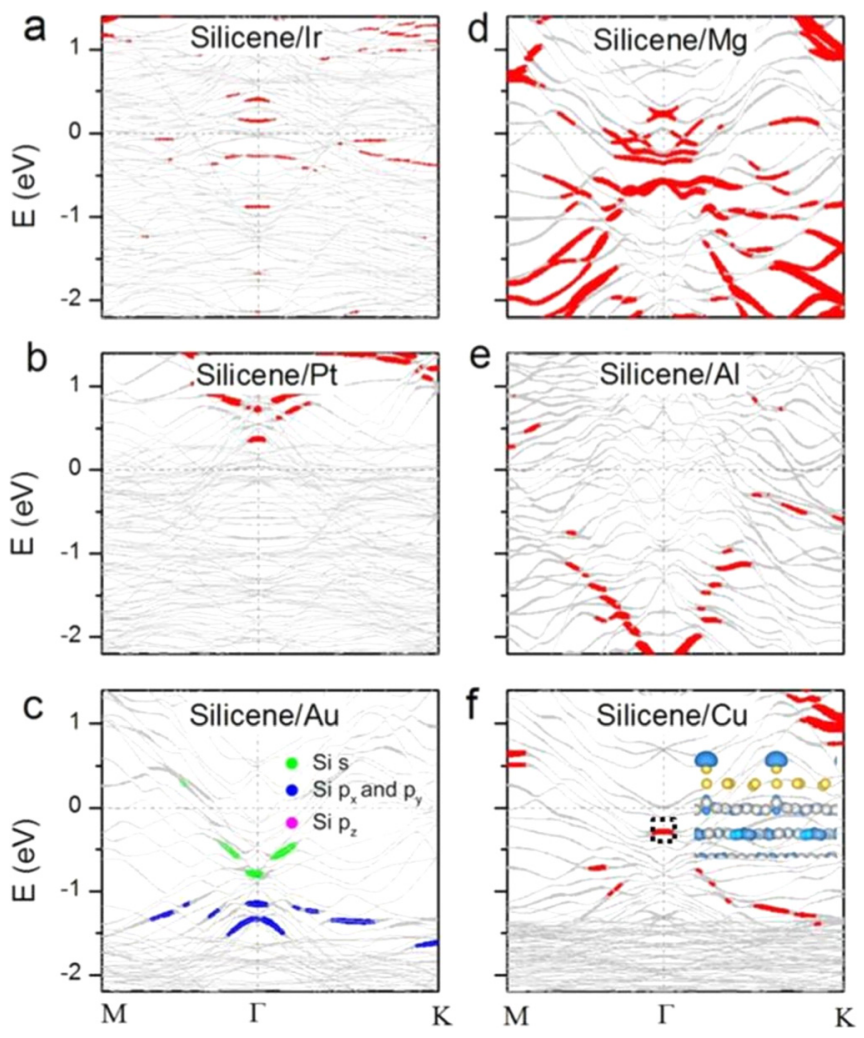

FIG. 15. Band structure of $(\sqrt{3} \times \sqrt{3})$ silicene on metallic substrates. The Fermi level is set to zero. The color indicates the Si states. The inset in (f) shows the electron density at the $\Gamma$ point inside the black dashed square, where the yellow and silver balls are $\mathrm{Si}$ and $\mathrm{Cu}$ atoms, respectively. Reprinted with permission from Quhe et al., Sci. Rep. 4, 5476 (2014). Copyright 2014 Macmillan Publishers Ltd: Nature. 
(a)

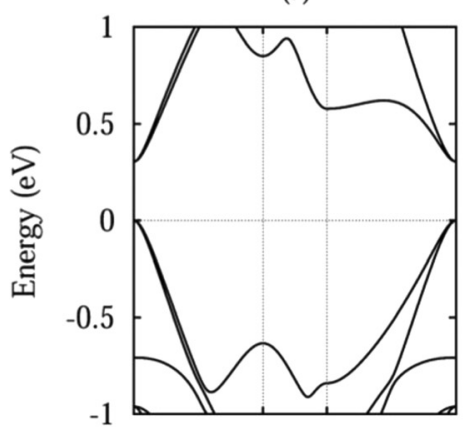

(d)

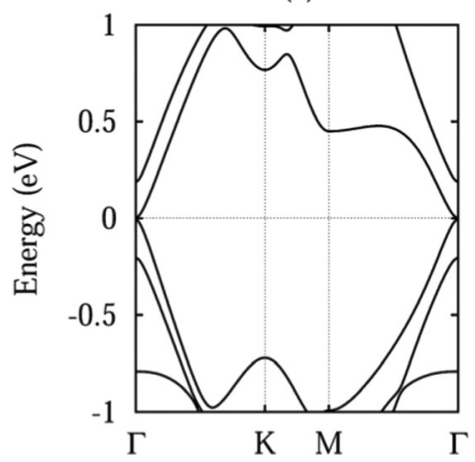

(b)

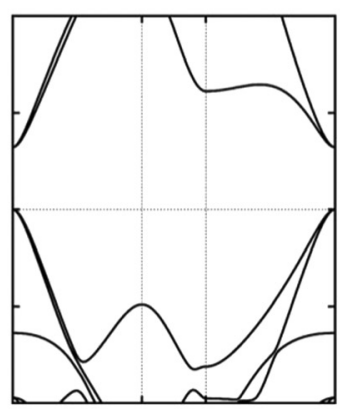

(e)

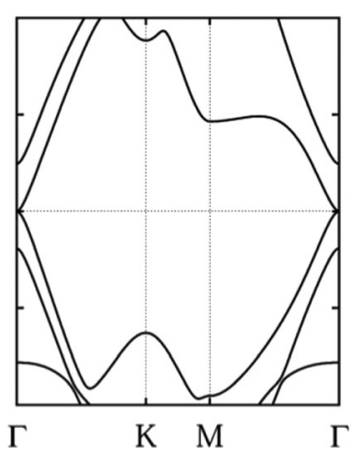

bulk $\mathrm{Si}$ is achieved. ${ }^{137}$ Low-buckled silicene on $\mathrm{MoS}_{2}$ substrate has a Dirac cone with a band gap of $70 \mathrm{meV}$, but only the high-buckled structure without Dirac cone can be prepared. ${ }^{138-141}$ Although the high-buckled structure has imaginary phonon frequency, it contradicts the experiments. ${ }^{142}$ Since the van der Waals interaction is stronger in $\mathrm{WSe}_{2}$ (large amount of electrons contributing to the dipole), lowbuckled silicene can be stabilized and shows a Dirac cone with a large band gap of $0.34 \mathrm{eV}$ (Figure 16). ${ }^{143}$ Although the band gap can be tuned by an external electric field or strain due to charge transfer within the silicene sheet, ${ }^{135,139}$ these methods are difficult to be realized in devices so that one has to rely on the effects of the substrate. Despite many studies, there is still no ideal substrate known that simultaneously avoids a large lattice mismatch, a too small band gap, a complicated control of terminal layers, and a too strong or too weak interaction of silicene.

\section{RELATIONSHIP TO SILICON AND OTHER ALLOTROPES}

Silicene is a hypothetical allotrope of silicon, since freestanding silicene has not yet been formed. Nevertheless, the fact that functionalized silicene has been made $^{3}$ (including the fact that silioxene could be viewed as a form of functionalized silicene ${ }^{144,145}$ ) and that silicene has been grown on a few substrates, ${ }^{11,94,97,146}$ it is widely expected that freestanding silicene could someday be achieved, possibly by peeling off the silicene from the substrate. ${ }^{147}$

A single silicene sheet is very much like a single (111) silicon plane, except that the vertical displacement of the $\mathrm{Si}$ atoms (perpendicular to the atomic plane) is about $0.45 \AA$ for silicene and it is $0.78 \AA$ for $\mathrm{Si}$. Labeling the bonding for Si to be pure $s p^{3}$, one could consider the bonding for silicene to be intermediate between $s p^{3}$ and the $s p^{2}$ of graphene.

(c)

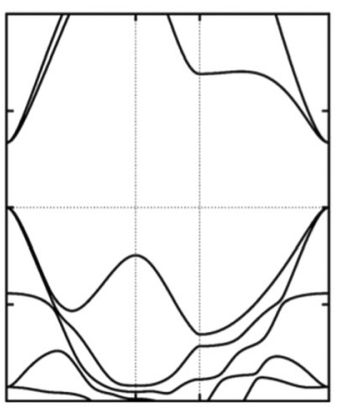

(f)

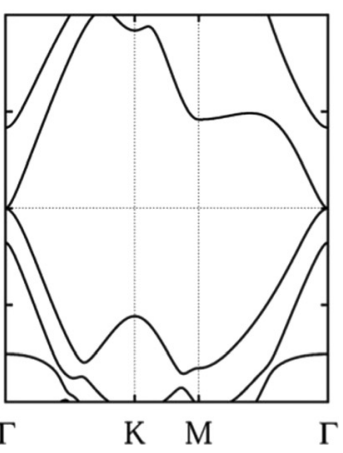

FIG. 16. Band structure of silicene on (a) and (d) S doped, (b) and (e) pristine, and (c) and (f) Te doped $\mathrm{WSe}_{2}$ substrate without lateral shift (upper row) and with a $1 / 3$ lateral shift (lower row). The Fermi level is set to zero. Reproduced with permission from J. Zhu and U. Schwingenschlögl, J. Mater. Chem. C 3, 3946 (2015). Copyright 2015 The Royal Society of Chemistry.
A more interesting problem is what happens if one were to have bilayers of silicene, then multilayers. Naively, one would expect bulk diamond $\mathrm{Si}$ to be recovered. Calculations and experiments are currently not all in agreement.

For the bilayer structure, first-principles MD calculations were carried out (at $300 \mathrm{~K}$ for $15 \mathrm{ps}$ ), starting with the ideal hexagonal structure and both $\mathrm{AA}$ and $\mathrm{AB}$ stacking. ${ }^{148}$ In both cases, the structure reconstructed into a $(2 \times 2)$ one. Other metastable structures could form but they transformed to the previous lower energy ones as the temperature was raised up to $1200 \mathrm{~K}$. On the other hand, Wen et al. ${ }^{149}$ did not find a reconstruction but simply two flat sheets in AA stacking for the bilayer silicene. The layer separation they obtained is $2.39 \AA$ compared to the $3.26 \AA$ for graphite. A bilayer was also studied by Fu et al.; ${ }^{150}$ they did not report reconstruction but instead the possibility of a stable form with the two layers shifted with respect to each other. On the other hand, Padilha and Pontes ${ }^{151}$ reported that the lowest energy conformation, labeled $\mathrm{AA}_{P}$, has flat silicene sheets (Fig. 17); they computed that there is an energy gain in forming $\sigma$ bonds rather than $\pi$ bonds.

Multilayer silicene has been reported to have been fabricated on Ag (111). ${ }^{108,110,152,153}$ Studies using LEED and STM have indicated that the $(\sqrt{3} \times \sqrt{3}) R 30^{\circ}$ phase is formed. ${ }^{152}$ More recent work using STM, however, point to a bulk diamond-like film with a $(\sqrt{3} \times \sqrt{3}) R 30^{\circ}$ honeycomb superstructure on the surface. ${ }^{154}$ The formation of multilayer silicene on silver has also been disputed theoretically. Thus, a new layered allotrope, silicite, has been proposed. ${ }^{155}$ Alternately, multilayers up to $30 \mathrm{ML}$ and beyond have been grown $^{156}$ and have been shown to be bulk Si (111) with the top layer having a $(\sqrt{3} \times \sqrt{3}) R 30^{\circ}$ honeycomb superstructure; the same structure has been confirmed by another group. ${ }^{157}$ From quasi-particle interference experiments, they also measured a Dirac cone which they attributed to the 

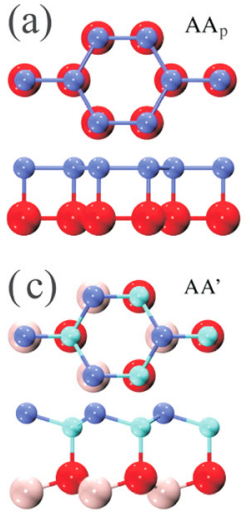

(e)

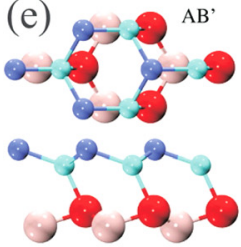

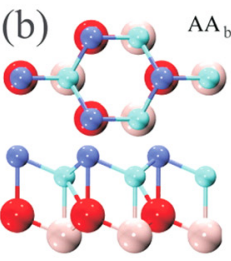

(d) $\rho-\rho^{\mathrm{AB}}$

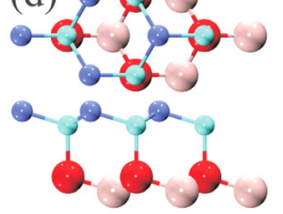

(f)

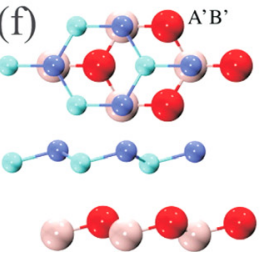

(g)

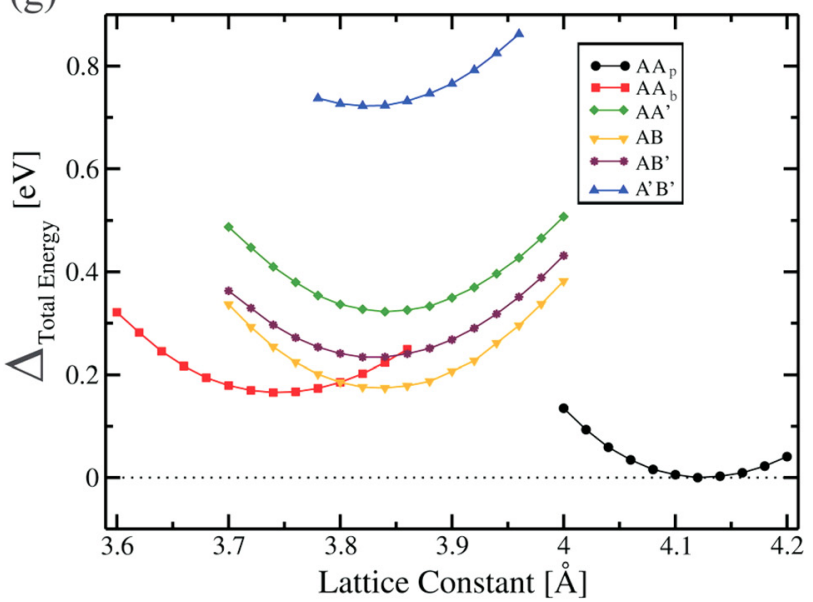

FIG. 17. Structure of bilayer silicene. Reprinted with permission from J. E. Padilha and R. B. Pontes, J. Phys. Chem. C 119, 3818 (2015). Copyright 2015 American Chemical Society.

hexagonal surface layer. Others have shown multilayer silicene to transform to the diamond structure. ${ }^{153}$

\section{DEVICES}

Potential applications of silicene have been considered theoretically, though only one has so far been realized experimentally. We provide an overview of the main device applications proposed.

\section{A. Thermoelectrics}

Zigzag silicene nanoribbons turn out to show interesting thermoelectric properties, including a low thermal conductivity, a high Seebeck coefficient, and therefore a high figure of merit. ${ }^{158}$ By tuning of the Fermi level via doping, a figure of merit of up to 3.5 can be achieved. ${ }^{159}$

\section{B. Field-effect transistors}

Silicene is compatible with the present silicon complementary metal-oxide-semiconductor (CMOS) technology in comparison to graphene. Application in field-effect transistors (FETs) requires a band gap of more than $0.4 \mathrm{eV}$ to achieve an adequate switching behavior and an on/off current ratio that makes it possible to obtain ultra high speed in the $\mathrm{THz}$ frequency range. Graphene supplies only an on/off ratio of $10^{4} .{ }^{160} \mathrm{Ir}, \mathrm{Cu}$, and $\mathrm{Pt}$ decoration of silicene with low concentration can open band gaps of about $0.2 \mathrm{eV}$ (p-doped, neutral n-doped). ${ }^{161}$ An on/off current ratio of $10^{3}$ has been achieved on a silicene p-i-n tunneling FET. A FET based on zigzag silicene nanoribbon $(\mathrm{N}=6)$ has been predicted to have a band gap of $0.44 \mathrm{eV}$ and an on/off current ratio of $10^{6} .{ }^{162} \mathrm{~A}$ much higher on/off current ratio of $10^{8}$ with a band gap of $0.45 \mathrm{eV}$ is predicted for silicene decorated with alkali metal atoms. ${ }^{70}$ Silicene nanoribbon can be used to realize a field-effect topological quantum transistor due to the quantized conductance changing from 2 to 0 in an electric field. ${ }^{163}$

\section{Gas adsorbent}

Two-dimensional materials are expected to be promising adsorbents for environmental gases, such as $\mathrm{NO}, \mathrm{SO}_{2}$, and $\mathrm{NH}_{3}$, because of the large surface-to-volume ratio. The binding energy of $\mathrm{NO}$ on silicene is calculated to be $0.35 \mathrm{eV}$ with a Si-N bond length of $2.1 \AA$, which reflects chemical adsorption. ${ }^{164-166}$ Two dissociation mechanisms for NO have been proposed. ${ }^{167}$ One is a direct breaking of the N-O bond with an energy barrier of $0.47 \mathrm{eV}$ and a subsequent formation of $\mathrm{Si}-\mathrm{O}$ and Si-N bonds. The other is the formation of a $(\mathrm{NO})_{2}$ dimer and subsequent decomposition into $\mathrm{N}_{2}$ and two $\mathrm{O}$ atoms bonded with $\mathrm{Si}$ (energy barrier of $0.45 \mathrm{eV}$ ). $\mathrm{CO}$ is predicted to be physically absorbed on silicene with a small binding energy of $0.10 \mathrm{eV}$ and accordingly a large bond length of $3.2 \AA .^{164,166}$ The energy barrier for dissociation on top of a vacancy is calculated to be $4.36 \mathrm{eV}$, as the distance between $\mathrm{CO}$ and the silicene sheet is reduced to $1.7 \AA{ }^{168}$ A large binding energy of more than $1.30 \mathrm{eV}$ is found for the adsorption of $\mathrm{NO}_{2}{ }^{164-166}$ This strong interaction is confirmed by a short $\mathrm{Si}$ $\mathrm{O}$ bond length of $1.7 \AA$. $\mathrm{NH}_{3}$ shows a binding energy of $0.6 \mathrm{eV}$ with a $\mathrm{Si}-\mathrm{N}$ bond length of $2.0 \AA$, ${ }^{164,165}$ whereas $\mathrm{CO}_{2}$ is subject to weak van der Waals interaction. A strong interaction is predicted for $\mathrm{SO}_{2}$ (binding energy of $1.07 \mathrm{eV}$ ). ${ }^{164} \mathrm{~B}$ and $\mathrm{N}$ substitutions as well as Stone-Wales defects turn out to enhance the interaction between gas molecules and silicene. ${ }^{164,166}$ While large binding energies enable freestanding silicene to reduce $\mathrm{NO}, \mathrm{NH}_{3}, \mathrm{SO}_{2}$, and $\mathrm{NO}_{2}$, it cannot absorb $\mathrm{CO}$ and $\mathrm{CO}_{2}$. This changes for Au decorated silicene, which absorbs $\mathrm{CO}$ with a binding energy of $0.87 \mathrm{eV}$ and oxidizes it into $\mathrm{CO}_{2}$ with a low energy barrier of $0.34 \mathrm{eV} .^{169}$

\section{Hydrogen storage}

In spite of being a clean energy source, ${ }^{170}$ the storage of $\mathrm{H}$ still is a major challenge and limits the field of applications. C-based nanowires and nanosheets have been proposed 
for hydrogen storage, but weak interaction due to $s p^{2}$ bonding leads to a low storage capacity. ${ }^{171,172}$ A moderate binding energy of $0.2-0.6 \mathrm{eV}$ with a storage capacity of more than $5.5 \mathrm{wt} . \%$ is required for technological purposes. ${ }^{173}$ Although the $s p^{2}-s p^{3}$ hybridization, in general, results in strong bonding with adsorbates, the binding energy of $\mathrm{H}_{2}$ on freestanding silicene is calculated to be only $0.11 \mathrm{eV}$. Metal atom decorated silicene is predicted to stabilize $\mathrm{H}_{2}$, as the binding energy ranges from 0.18 to $0.37 \mathrm{eV}$ for $\mathrm{Li}, \mathrm{Na}, \mathrm{K}$, and $\mathrm{Ca}$ decoration. ${ }^{73,173-176}$ The number of $\mathrm{H}_{2}$ molecules that can be absorbed by one adatom is $4,4,5$, and 6 , leading to a storage capacity of $6.3,5.4,6.1$, and $7.3 \mathrm{wt}$. \%. Although $\mathrm{Mg}$ can bond $6 \mathrm{H}_{2}$ molecules with a binding energy of more than $0.15 \mathrm{eV}$, and thus reaches a high capacity of $8.1 \mathrm{wt} . \%$, a biaxial tensile strain of $10 \%$ has to be applied to prevent $\mathrm{Mg}$ from forming clusters. ${ }^{177}$ For Sc, Ti, and V decorations, the binding energy increases to more than $0.4 \mathrm{eV}$, but the capacities decrease to less than $5 \mathrm{wt} . \%{ }^{73}$ The binding energy is affected by an electric field because charge transfer facilitates the storage and release processes. ${ }^{173}$ Not only the ionic radius but also the mass of the adatoms needs to be considered to achieve a high $\mathrm{H}_{2}$ storage capacity.

\section{E. Li-ion batteries}

Li-ion batteries nowadays are widely used in portable devices and vehicles because of the high energy density. ${ }^{178}$ Besides the large surface-to-volume ratio of twodimensional materials and the resulting high $\mathrm{Li}$ specific capacity, a high mobility of the charge carriers can be realized by linearly dispersing $\pi$ and $\pi^{*}$ bands. A Li storage capacity of $540 \mathrm{~mA} \mathrm{~h} / \mathrm{g}$ is predicted for graphene (commercial graphite anode: $372 \mathrm{~mA} \mathrm{~h} / \mathrm{g}$ ). ${ }^{179}$ However, the energy barriers for Li migration on the surface of $10 \mathrm{eV}$ for the pristine graphene and $1.34 \mathrm{eV}$ for the defective graphene with double vacancies are much higher than in graphite $(0.22 \mathrm{eV})$, which prohibits the high cycling rate. ${ }^{180}$ Pristine silicene achieves high Li capacities of $954 \mathrm{~mA} \mathrm{~h} / \mathrm{g}$ for a monolayer and $715 \mathrm{~mA} \mathrm{h/g}$ for a bilayer with a lower diffusion barrier of $0.23 \mathrm{eV} .{ }^{181} \mathrm{~B}$ substitution at the $\mathrm{Si}$ site turns out to improve the capacity to 1410 and $846 \mathrm{~mA} \mathrm{~h} / \mathrm{g}$ for the monolayer and bilayer, respectively, without affecting the diffusion barrier. ${ }^{182}$ The energy barrier for diffusion $(1.59 \mathrm{eV}$ in pristine silicene) is predicted to be reduced to $0.88,0.86$, and $0.05 \mathrm{eV}$ in the presence of single vacancies, Stone-Wales defects, and divacancies, respectively. ${ }^{75}$ These defects slightly enhance the on-surface diffusion barrier to $0.3 \mathrm{eV}$. The Li storage capacity is adversely affected by any substrate. Layered substrates with small atomic masses and moderate interaction with silicene are preferred for usage as electrode material in Li-ion batteries.

\section{SUMMARY}

The study of silicene is still very active, and we hope to have conveyed the fact that many of the results are still being debated. Most of the papers to date have been theoretical work due to the difficulty in obtaining silicene samples. Whether it is possible to obtain a freestanding two- dimensional allotrope of silicon has not been proven but this is certainly a very exciting search.

\section{ACKNOWLEDGMENTS}

L.C.L.Y.V. would like to thank The Citadel Foundation and the Traubert Chair for partially funding this work, and the NSF for funding that led to our research into silicene. Research reported in this publication was supported by funding from the King Abdullah University of Science and Technology (KAUST).

${ }^{1}$ G. G. Guzmán-Verri and L. C. Lew Yan Voon, Phys. Rev. B 76, 075131 (2007).

${ }^{2}$ X. Yang and J. Ni, Phys. Rev. B 72, 195426 (2005).

${ }^{3}$ H. Nakano, T. Mitsuoka, M. Harada, K. Horibuchi, H. Nozaki, N. Takahashi, T. Nonaka, Y. Seno, and H. Nakamura, Angew. Chem. 118, 6451 (2006).

${ }^{4}$ K. Takeda and K. Shiraishi, Phys. Rev. B 50, 14916 (1994).

${ }^{5}$ E. Durgun, S. Tongay, and S. Ciraci, Phys. Rev. B 72, 075420 (2005).

${ }^{6}$ A. Kara, C. Léandri, M. E. Dávila, P. de Padova, B. Ealet, H. Oughaddou, B. Aufray, and G. L. Lay, J. Supercond. Novel Magn. 22, 259 (2009).

${ }^{7}$ D. Chiappe, C. Grazianetti, G. Tallarida, M. Fanciulli, and A. Molle, Adv. Mater. 24, 5088 (2012).

${ }^{8}$ B. Feng, Z. Ding, S. Meng, Y. Yao, X. He, P. Cheng, L. Chen, and K. Wu, Nano Lett. 12, 3507 (2012).

${ }^{9}$ H. Jamgotchian, Y. Colignon, N. Hamzaoui, B. Ealet, J. Y. Hoarau, B. Aufray, and J. P. Bibérian, J. Phys.: Condens. Matter 24, 172001 (2012).

${ }^{10}$ C.-L. Lin, R. Arafune, K. Kawahara, N. Tsukahara, E. Minamitani, Y. Kim, N. Takagi, and M. Kawai, Appl. Phys. Express 5, 045802 (2012).

${ }^{11}$ P. Vogt, P. De Padova, C. Quaresima, J. Avila, E. Frantzeskakis, M. C. Asensio, A. Resta, B. Ealet, and G. Le Lay, Phys. Rev. Lett. 108, 155501 (2012).

${ }^{12}$ S. Cahangirov, M. Topsakal, E. Aktürk, H. Şahin, and S. Ciraci, Phys. Rev. Lett. 102, 236804 (2009).

${ }^{13}$ D. Kaltsas and L. Tsetseris, Phys. Chem. Chem. Phys. 15, 9710 (2013).

${ }^{14}$ T. P. Kaloni, Y. C. Cheng, and U. Schwingenschlögl, J. Appl. Phys. 113, 104305 (2013).

${ }^{15}$ Q. Peng, X. Wen, and S. De, RSC Adv. 3, 13772 (2013).

${ }^{16}$ Z. Ni, Q. Liu, K. Tang, J. Zheng, J. Zhou, R. Qin, Z. Gao, D. Yu, and J. Lu, Nano Lett. 12, 113 (2012).

${ }^{17}$ H. Şahin, S. Cahangirov, M. Topsakal, E. Bekaroglu, E. Akturk, R. T. Senger, and S. Ciraci, Phys. Rev. B 80, 155453 (2009).

${ }^{18}$ G. Liu, M. S. Wu, C. Y. Ouyang, and B. Xu, EPL (Europhys. Lett.) 99, 17010 (2012).

${ }^{19}$ R. Qin, C.-H. Wang, W. Zhu, and Y. Zhang, AIP Adv. 2, 022159 (2012).

${ }^{20}$ H. Zhao, Phys. Lett. A 376, 3546 (2012).

${ }^{21}$ R. Qin, W. Zhu, Y. Zhang, and X. Deng, Nanoscale Res. Lett. 9, 521 (2014).

${ }^{22}$ C. Yang, Z. Yu, P. Lu, Y. Liu, H. Ye, and T. Gao, Comput. Mater. Sci. 95, 420 (2014).

${ }^{23}$ B. Wang, J. Wu, X. Gu, H. Yin, Y. Wei, R. Yang, and M. Dresselhaus, Appl. Phys. Lett. 104, 081902 (2014).

${ }^{24}$ C.-C. Liu, W. Feng, and Y. Yao, Phys. Rev. Lett. 107, 076802 (2011).

${ }^{25}$ C. L. Kane and E. J. Mele, Phys. Rev. Lett. 95, 226801 (2005).

${ }^{26}$ M. Ezawa, Phys. Rev. Lett. 109, 055502 (2012).

${ }^{27}$ Y. Wang and Y. Ding, Solid State Commun. 155, 6 (2013).

${ }^{28}$ A. Durajski, D. Szcześniak, and R. Szcześniak, Solid State Commun. 200, 17 (2014).

${ }^{29}$ B. Mohan, A. Kumar, and P. Ahluwalia, Physica E 61, 40 (2014).

${ }^{30}$ L. C. Lew Yan Voon, A. Lopez-Bezanilla, J. Wang, Y. Zhang, and M. Willatzen, New J. Phys. 17, 025004 (2015).

${ }^{31}$ N. D. Drummond, V. Zólyomi, and V. I. Fal'ko, Phys. Rev. B 85, 075423 (2012).

${ }^{32}$ V. Vargiamidis, P. Vasilopoulos, and G.-Q. Hai, J. Phys.: Condens. Matter 26, 345303 (2014).

${ }^{33}$ M. Syaputra, S. A. Wella, T. D. K. Wungu, A. Purqon, and Suprijadi, AIP Conf. Proc. 1677, 080012 (2015).

${ }^{34}$ Z.-G. Shao, X.-S. Ye, L. Yang, and C.-L. Wang, J. Appl. Phys. 114, 093712 (2013).

${ }^{35}$ G. Berdiyorov and F. Peeters, RSC Adv. 4, 1133 (2014).

${ }^{36}$ H.-P. Li and R.-Q. Zhang, EPL (Europhys. Lett.) 99, 36001 (2012). 
${ }^{37}$ A. A. Balandin, S. Ghosh, W. Bao, I. Calizo, D. Teweldebrhan, F. Miao, and C. N. Lau, Nano Lett. 8, 902 (2008).

${ }^{38}$ M. Kamatagi, J. Elliott, N. Sankeshwar, and A. Lindsay Greer, in Physics of Semiconductor Devices, Environmental Science and Engineering, edited by V. K. Jain and A. Verma (Springer International Publishing, 2014), pp. 617-619, ISBN: 978-3-319-03001-2.

${ }^{39}$ K. Yang, S. Cahangirov, A. Cantarero, A. Rubio, and R. D'Agosta, Phys. Rev. B 89, 125403 (2014).

${ }^{40}$ Y. Jing, Y. Sun, H. Niu, and J. Shen, Phys. Status Solidi B 250, 1505 (2013).

${ }^{41}$ M. Topsakal and S. Ciraci, Phys. Rev. B 81, 024107 (2010).

${ }^{42}$ Y. Borensztein, G. Prévot, and L. Masson, Phys. Rev. B 89, 245410 (2014).

${ }^{43}$ P. D. Padova, C. Quaresima, B. Olivieri, P. Perfetti, and G. L. Lay, Appl. Phys. Lett. 98, 081909 (2011).

${ }^{44}$ S. Kaneko, H. Tsuchiya, Y. Kamakura, N. Mori, and M. Ogawa, Appl. Phys. Express 7, 035102 (2014)

${ }^{45}$ Y. Ding and J. Ni, Appl. Phys. Lett. 95, 083115 (2009).

${ }^{46}$ V. Barone, O. Hod, and G. E. Scuseria, Nano Lett. 6, 2748 (2006).

${ }^{47}$ Y.-L. Song, S. Zhang, D.-B. Lu, H.-R. Xu, Z. Wang, Y. Zhang, and Z.W. Lu, Eur. Phys. J. B 86, 1 (2013).

${ }^{48}$ Y. Liang, V. Wang, H. Mizuseki, and Y. Kawazoe, J. Phys.: Condens. Matter 24, 455302 (2012)

${ }^{49}$ J. Chen, X.-F. Wang, P. Vasilopoulos, A.-B. Chen, and J.-C. Wu, ChemPhysChem 15, 2701 (2014).

${ }^{50}$ N. B. Le, T. D. Huan, and L. M. Woods, Phys. Rev. Appl. 1, 054002 (2014).

${ }^{51}$ Y.-L. Song, J.-M. Zhang, D.-B. Lu, and K.-W. Xu, Physica E 56, 205 (2014).

${ }^{52}$ Y.-L. Song, Y. Zhang, J.-M. Zhang, D.-B. Lu, and K.-W. Xu, Eur. Phys. J. B 79, 197 (2011).

${ }^{53}$ Y.-L. Song, J.-M. Zhang, D.-B. Lu, and K.-W. Xu, Physica E 53, 173 (2013).

${ }^{54}$ T. H. Osborn, A. A. Farajian, O. V. Pupysheva, R. S. Aga, and L. Lew Yan Voon, Chem. Phys. Lett. 511, 101 (2011).

${ }^{55}$ L. C. Lew Yan Voon, E. Sandberg, R. S. Aga, and A. A. Farajian, Appl. Phys. Lett. 97, 163114 (2010).

${ }^{56}$ M. Houssa, E. Scalise, K. Sankaran, G. Pourtois, V. V. Afanas'ev, and A. Stesmans, Appl. Phys. Lett. 98, 223107 (2011).

${ }^{57}$ G. Cao, Y. Zhang, and J. Cao, Phys. Lett. A 379, 1475 (2015).

${ }^{58}$ B. van den Broek, M. Houssa, E. Scalise, G. Pourtois, V. Afanas'ev, and A. Stesmans, Appl. Surf. Sci. 291, 104 (2013).

${ }^{59}$ B. Huang, H. J. Xiang, and S.-H. Wei, Phys. Rev. Lett. 111, 145502 (2013)

${ }^{60}$ B. Huang, H.-X. Deng, H. Lee, M. Yoon, B. G. Sumpter, F. Liu, S. C. Smith, and S.-H. Wei, Phys. Rev. X 4, 021029 (2014).

${ }^{61}$ W. Rui, W. Shaofeng, and W. Xiaozhi, J. Appl. Phys. 116, 024303 (2014).

${ }^{62}$ W. C. Wu, Z. M. Ao, C. H. Yang, S. Li, G. X. Wang, C. M. Li, and S. Li, J. Mater. Chem. C 3, 2593 (2015).

${ }^{63}$ W. Wu, Z. Ao, T. Wang, C. Li, and S. Li, Phys. Chem. Chem. Phys. 16, 16588 (2014).

${ }^{64}$ G. Liu, X. L. Lei, M. S. Wu, B. Xu, and C. Y. Ouyang, EPL (Europhys. Lett.) 106, 47001 (2014).

${ }^{65}$ Y. Wang and Y. Ding, Phys. Status Solidi RRL 7, 410 (2013).

${ }^{66}$ V. O. Özęlik and S. Ciraci, J. Phys. Chem. C 117, 26305 (2013).

${ }^{67}$ G. Liu, X. L. Lei, M. S. Wu, B. Xu, and C. Y. Ouyang, J. Phys.: Condens. Matter 26, 355007 (2014).

${ }^{68}$ N. Gao, W. Zheng, and Q. Jiang, Phys. Chem. Chem. Phys. 14, 257 (2012)

${ }^{69}$ N. Wang, H. Guo, Y.-J. Liu, J.-X. Zhao, Q.-H. Cai, and X.-Z. Wang, Physica E 73, 21 (2015)

${ }^{70}$ R. Quhe, R. Fei, Q. Liu, J. Zheng, H. Li, C. Xu, Z. Ni, Y. Wang, D. Yu, Z. Gao, and J. Lu, Sci. Rep. 2, 853 (2012).

${ }^{71}$ H. Sahin and F. M. Peeters, Phys. Rev. B 87, 085423 (2013).

${ }^{72}$ X. Lin and J. Ni, Phys. Rev. B 86, 075440 (2012).

${ }^{73}$ Y. Wang, R. Zheng, H. Gao, J. Zhang, B. Xu, Q. Sun, and Y. Jia, Int. J. Hydrogen Energy 39, 14027 (2014).

${ }^{74}$ T. H. Osborn and A. A. Farajian, J. Phys. Chem. C 116, 22916 (2012).

${ }^{75}$ J. Setiadi, M. D. Arnold, and M. J. Ford, ACS Appl. Mater. Interfaces 5, 10690 (2013).

${ }^{76}$ X. Guo, P. Guo, J. Zheng, L. Cao, and P. Zhao, Appl. Surf. Sci. 341, 69 (2015).
${ }^{77}$ Y. Lee, K.-H. Yun, S. B. Cho, and Y.-C. Chung, ChemPhysChem 15, 4095 (2014).

${ }^{78}$ V. Q. Bui, T.-T. Pham, H.-V. S. Nguyen, and H. M. Le, J. Phys. Chem. C 117, 23364 (2013).

${ }^{79}$ N. Y. Dzade, K. O. Obodo, S. K. Adjokatse, A. C. Ashu, E. Amankwah, C. D. Atiso, A. A. Bello, E. Igumbor, S. B. Nzabarinda, J. T. Obodo, A. O. Ogbuu, O. E. Femi, J. O. Udeigwe, and U. V. Waghmare, J. Phys.: Condens. Matter 22, 375502 (2010).

${ }^{80}$ H. Johll, M. D. K. Lee, S. P. N. Ng, H. C. Kang, and E. S. Tok, Sci. Rep. 4, 7594 (2014).

${ }^{81}$ T. P. Kaloni, S. Gangopadhyay, N. Singh, B. Jones, and U. Schwingenschlögl, Phys. Rev. B 88, 235418 (2013).

${ }^{82}$ J. Zhang, B. Zhao, and Z. Yang, Phys. Rev. B 88, 165422 (2013).

${ }^{83}$ T. P. Kaloni and U. Schwingenschlögl, Phys. Status Solidi RRL 8, 685 (2014).

${ }^{84}$ F. Ersan, Ö. Arslanalp, G. Gökoğlu, and E. Aktürk, Appl. Surf. Sci. 311, 9 (2014).

${ }^{85}$ J. Dai and X. C. Zeng, Phys. Chem. Chem. Phys. 17, 17957 (2015).

${ }^{86}$ P. A. Denis, Phys. Chem. Chem. Phys. 17, 5393 (2015).

${ }^{87}$ T. Zhao, S. Zhang, Q. Wang, Y. Kawazoe, and P. Jena, Phys. Chem. Chem. Phys. 16, 22979 (2014)

${ }^{88}$ T. P. Kaloni, G. Schreckenbach, and M. S. Freund, J. Phys. Chem. C 118, 23361 (2014).

${ }^{89}$ K. Mondal, C. Kamal, A. Banerjee, A. Chakrabarti, and T. K. Ghanty, J. Phys. Chem. C 119, 3192 (2015)

${ }^{90}$ J. Gao and J. Zhao, Sci. Rep. 2, 861 (2012).

${ }^{91}$ Z.-X. Guo, S. Furuya, J.-I. Iwata, and A. Oshiyama, Phys. Rev. B 87, 235435 (2013).

${ }^{92}$ Y.-P. Wang and H.-P. Cheng, Phys. Rev. B 87, 245430 (2013).

${ }^{93}$ B. Lalmi, H. Oughaddou, H. Enriquez, A. Kara, S. Vizzini, B. Ealet, and B. Aufray, Appl. Phys. Lett. 97, 223109 (2010).

${ }^{94}$ L. Meng, Y. Wang, L. Zhang, S. Du, R. Wu, L. Li, Y. Zhang, G. Li, H. Zhou, W. A. Hofer, and H.-J. Gao, Nano Lett. 13, 685 (2013).

${ }^{95}$ T. Aizawa, S. Suehara, and S. Otani, J. Phys.: Condens. Matter 27, 305002 (2015).

${ }^{96}$ C.-C. Lee, A. Fleurence, Y. Yamada-Takamura, T. Ozaki, and R. Friedlein, Phys. Rev. B 90, 075422 (2014).

${ }^{97}$ T. Aizawa, S. Suehara, and S. Otani, J. Phys. Chem. C 118, 23049 (2015).

${ }^{98}$ D. Kaltsas, L. Tsetseris, and A. Dimoulas, J. Phys.: Condens. Matter 24, 442001 (2012)

${ }^{99}$ R. Stephan, M.-C. Hanf, and P. Sonnet, J. Phys.: Condens. Matter 27, 015002 (2015).

${ }^{100}$ Y. Yuan, R. Quhe, J. Zheng, Y. Wang, Z. Ni, J. Shi, and J. Lu, Physica E $\mathbf{5 8}, 38(2014)$

${ }^{101}$ G. Prévot, R. Bernard, H. Cruguel, and Y. Borensztein, Appl. Phys. Lett. 105, 213106 (2014).

${ }^{102}$ J. Sone, T. Yamagami, Y. Aoki, K. Nakatsuji, and H. Hirayama, New J. Phys. 16, 095004 (2014)

${ }^{103}$ P. Moras, T. O. Mentes, P. M. Sheverdyaeva, A. Locatelli, and C. Carbone, J. Phys.: Condens. Matter 26, 185001 (2014).

${ }^{104}$ R. Arafune, C.-L. Lin, K. Kawahara, N. Tsukahara, E. Minamitani, Y. Kim, N. Takagi, and M. Kawai, Surf. Sci. 608, 297 (2013).

${ }^{105}$ C. Grazianetti, D. Chiappe, E. Cinquanta, M. Fanciulli, and A. Molle, J. Phys.: Condens. Matter 27, 255005 (2015).

${ }^{106}$ D. Kaltsas, L. Tsetseris, and A. Dimoulas, Appl. Surf. Sci. 291, 93 (2013).

${ }^{107}$ A. Acun, B. Poelsema, H. J. W. Zandvliet, and R. van Gastel, Appl. Phys. Lett. 103, 263119 (2013).

${ }^{108}$ N. W. Johnson, D. Muir, E. Z. Kurmaev, and A. Moewes, Adv. Funct. Mater. 25, 4083 (2015).

${ }^{109}$ Z.-X. Guo and A. Oshiyama, Phys. Rev. B 89, 155418 (2014).

${ }^{110}$ P. Vogt, P. Capiod, M. Berthe, A. Resta, P. De Padova, T. Bruhn, G. Le Lay, and B. Grandidier, Appl. Phys. Lett. 104, 021602 (2014).

${ }^{111}$ P. De Padova, J. Avila, A. Resta, I. Razado-Colambo, C. Quaresima, C. Ottaviani, B. Olivieri, T. Bruhn, P. Vogt, M. C. Asensio, and G. Le Lay, J. Phys.: Condens. Matter 25, 382202 (2013).

${ }^{112}$ M. X. Chen and M. Weinert, Nano Lett. 14, 5189 (2014).

${ }^{113}$ P. Pflugradt, L. Matthes, and F. Bechstedt, Phys. Rev. B 89, 205428 (2014).

${ }^{114}$ T. Morishita, M. J. S. Spencer, S. Kawamoto, and I. K. Snook, J. Phys. Chem. C 117, 22142 (2013)

${ }^{115}$ R. Quhe, Y. Yuan, J. Zheng, Y. Wang, Z. Ni, J. Shi, D. Yu, J. Yang, and J. Lu, Sci. Rep. 4, 5476 (2014). 
${ }^{116}$ A. Podsiadly-Paszkowska and M. Krawiec, Phys. Chem. Chem. Phys. 17, 2246 (2015).

${ }^{117}$ S. Si Li, C.-W. Zhang, S.-S. Yan, S.-J. Hu, W.-X. Ji, P.-J. Wang, and P. Li, J. Phys.: Condens. Matter 26, 395003 (2014).

${ }^{118}$ A. Bhattacharya, S. Bhattacharya, and G. P. Das, Appl. Phys. Lett. 103, 123113 (2013).

${ }^{119}$ M. Houssa, G. Pourtois, M. M. Heyns, V. V. Afanas'ev, and A. Stesmans, J. Electrochem. Soc. 158, H107 (2011).

${ }^{120}$ Y. Ding and Y. Wang, Appl. Phys. Lett. 103, 043114 (2013).

${ }^{121}$ F. Filippone, J. Phys.: Condens. Matter 26, 395009 (2014).

${ }^{122}$ R.-W. Zhang, C.-W. Zhang, W.-X. Ji, M.-J. Ren, F. Li, and M. Yuan, Mater. Chem. Phys. 156, 89 (2015).

${ }^{123}$ R.-W. Zhang, C.-W. Zhang, W.-X. Ji, S.-J. Hu, S.-S. Yan, S.-S. Li, P. Li, P.-J. Wang, and Y.-S. Liu, J. Phys. Chem. C 118, 25278 (2014).

${ }^{124}$ N. Gao, J. Li, and Q. Jiang, Chem. Phys. Lett. 592, 222 (2014).

${ }^{125}$ H. Liu, J. Gao, and J. Zhao, J. Phys. Chem. C 117, 10353 (2013).

${ }^{126}$ S. Kokott, L. Matthes, and F. Bechstedt, Phys. Status Solidi RRL 7, 538 (2013).

${ }^{127}$ P. Pflugradt, L. Matthes, and F. Bechstedt, New J. Phys. 16, 075004 (2014).

${ }^{128}$ S. Kokott, P. Pflugradt, L. Matthes, and F. Bechstedt, J. Phys.: Condens. Matter 26, 185002 (2014).

${ }^{129}$ K. S. Novoselov, A. K. Geim, S. V. Morozov, D. Jiang, Y. Zhang, S. V. Dubonos, I. V. Grigorieva, and A. A. Firsov, Science 306, 666 (2004).

${ }^{130}$ X. Li, X. Hao, M. Zhao, Y. Wu, J. Yang, Y. Tian, and G. Qian, Adv. Mater. 25, 2200 (2013).

${ }^{131}$ G. R. Berdiyorov, M. Neek-Amal, F. M. Peeters, and A. C. T. van Duin, Phys. Rev. B 89, 024107 (2014).

${ }^{132}$ Y. Cai, C.-P. Chuu, C. M. Wei, and M. Y. Chou, Phys. Rev. B 88, 245408 (2013).

${ }^{133}$ M. Neek-Amal, A. Sadeghi, G. R. Berdiyorov, and F. M. Peeters, Appl. Phys. Lett. 103, 261904 (2013).

${ }^{134}$ L. Li, X. Wang, X. Zhao, and M. Zhao, Phys. Lett. A 377, 2628 (2013).

${ }^{135}$ H. Zhao, C. Zhang, S. Li, W. Ji, and P. Wang, J. Appl. Phys. 117, 085306 (2015).

${ }^{136}$ J. Zhu and U. Schwingenschlögl, ACS Appl. Mater. Interfaces 6, 11675 (2014).

${ }^{137}$ J. Zhu and U. Schwingenschlögl, ACS Appl. Mater. Interfaces 6, 19242 (2014).

${ }^{138}$ L. Li and M. Zhao, J. Phys. Chem. C 118, 19129 (2014).

${ }^{139}$ N. Gao, J. C. Li, and Q. Jiang, Phys. Chem. Chem. Phys. 16, 11673 (2014).

${ }^{140}$ E. Scalise, M. Houssa, E. Cinquanta, C. Grazianetti, B. van den Broek, G. Pourtois, A. Stesmans, M. Fanciulli, and A. Molle, 2D Mater. 1, 011010 (2014).

${ }^{141}$ D. Chiappe, E. Scalise, E. Cinquanta, C. Grazianetti, B. van den Broek, M. Fanciulli, M. Houssa, and A. Molle, Adv. Mater. 26, 2096 (2014).

${ }^{142}$ N. J. Roome and J. D. Carey, ACS Appl. Mater. Interfaces 6, 7743 (2014).

${ }^{143}$ J. Zhu and U. Schwingenschlögl, J. Mater. Chem. C 3, 3946 (2015).

${ }^{144}$ H. Nakano, M. Ishii, and H. Nakamura, Chem. Commun. 2005, 2945 (2005).

${ }^{145}$ Y. Sugiyama, H. Okamoto, T. Mitsuoka, T. Morikawa, K. Nakanishi, T. Ohta, and H. Nakano, J. Am. Chem. Soc. 132, 5946 (2010).

${ }^{146}$ A. Fleurence, R. Friedlein, T. Ozaki, H. Kawai, Y. Wang, and Y. Yamada-Takamura, Phys. Rev. Lett. 108, 245501 (2012).

${ }^{147}$ Z. Qin, Z. Xu, and M. Buehler, ASME J. Appl. Mech. 82, 101003 (2015).

${ }^{148}$ T. Morishita, M. J. Spencer, S. P. Russo, I. K. Snook, and M. Mikami, Chem. Phys. Lett. 506, 221 (2011).

${ }^{149}$ X.-D. Wen, T. J. Cahill, and R. Hoffmann, Chem.: Eur. J. 16, 6555 (2010).
${ }^{150}$ H. Fu, J. Zhang, Z. Ding, H. Li, and S. Meng, Appl. Phys. Lett. 104, 131904 (2014).

${ }^{151}$ J. E. Padilha and R. B. Pontes, J. Phys. Chem. C 119, 3818 (2015).

${ }^{152}$ E. Salomon, R. E. Ajjouri, G. L. Lay, and T. Angot, J. Phys.: Condens. Matter 26, 185003 (2014).

${ }^{153} \mathrm{H}$. Fu, L. Chen, J. Chen, J. Qiu, Z. Ding, J. Zhang, K. Wu, H. Li, and S. Meng, Nanoscale 7, 15880 (2015).

${ }^{154}$ J. Chen, Y. Du, Z. Li, W. Li, B. Feng, J. Qiu, P. Cheng, S. X. Dou, L. Chen, and K. Wu, Sci. Rep. 5, 13590 (2015).

${ }^{155}$ S. Cahangirov, V. O. Özçelik, A. Rubio, and S. Ciraci, Phys. Rev. B 90, 085426 (2014).

${ }^{156}$ J. Chen, W. Li, B. Feng, P. Cheng, J. Qiu, L. Chen, and K. Wu, e-print arXiv: 1405.7534

${ }^{157}$ T. Shirai, T. Shirasawa, T. Hirahara, N. Fukui, T. Takahashi, and S. Hasegawa, Phys. Rev. B 89, 241403 (2014).

${ }^{158}$ K. Zberecki, M. Wierzbicki, J. Barnaś, and R. Swirkowicz, Phys. Rev. B 88, 115404 (2013).

${ }^{159}$ H. Sadeghi, S. Sangtarash, and C. Lambert, Sci. Rep. 5, 9514 (2015).

${ }^{160} \mathrm{~F}$. Schwierz, Nat. Nano 5, 487 (2010).

${ }^{161}$ Z. Ni, H. Zhong, X. Jiang, R. Quhe, G. Luo, Y. Wang, M. Ye, J. Yang, J. Shi, and J. Lu, Nanoscale 6, 7609 (2014).

${ }^{162} \mathrm{H}$. Li, L. Wang, Q. Liu, J. Zheng, W.-N. Mei, Z. Gao, J. Shi, and J. Lu, Eur. Phys. J. B 85, 274 (2012).

${ }^{163}$ M. Ezawa, Appl. Phys. Lett. 102, 172103 (2013).

${ }^{164}$ J.-W. Feng, Y.-J. Liu, H.-X. Wang, J.-X. Zhao, Q.-H. Cai, and X.-Z. Wang, Comput. Mater. Sci. 87, 218 (2014).

${ }^{165}$ W. Hu, N. Xia, X. Wu, Z. Li, and J. Yang, Phys. Chem. Chem. Phys. 16, 6957 (2014).

${ }^{166}$ J. Prasongkit, R. G. Amorim, S. Chakraborty, R. Ahuja, R. H. Scheicher, and V. Amornkitbamrung, J. Phys. Chem. C 119, 16934 (2015).

${ }^{167}$ X. Xu, J. Li, X. Zhang, H. Xu, Z.-F. Ke, and C. Zhao, RSC Adv. 5, 22135 (2015).

${ }^{168}$ H. H. Gürel, V. O. Özęlik, and S. Ciraci, J. Phys. Chem. C 118, 27574 (2014).

${ }^{169}$ C. Li, S. Yang, S.-S. Li, J.-B. Xia, and J. Li, J. Phys. Chem. C 117, 483 (2013).

${ }^{170}$ W. Lubitz and W. Tumas, Chem. Rev. 107, 3900 (2007).

${ }^{171}$ W. H. Shin, S. H. Yang, W. A. Goddard, and J. K. Kang, Appl. Phys. Lett. 88, 053111 (2006).

${ }^{172}$ T. Yildirim and S. Ciraci, Phys. Rev. Lett. 94, 175501 (2005).

${ }^{173}$ E. H. Song, S. H. Yoo, J. J. Kim, S. W. Lai, Q. Jiang, and S. O. Cho, Phys. Chem. Chem. Phys. 16, 23985 (2014).

${ }^{174}$ T. Hussain, T. Kaewmaraya, S. Chakraborty, and R. Ahuja, Phys. Chem. Chem. Phys. 15, 18900 (2013).

${ }^{175} \mathrm{~F}$. Li, C.-W. Zhang, H.-X. Luan, and P.-J. Wang, J. Nanopart. Res. 15, 1972 (2013).

${ }^{176}$ J. Wang, J. Li, S.-S. Li, and Y. Liu, J. Appl. Phys. 114, 124309 (2013).

${ }^{177}$ T. Hussain, S. Chakraborty, A. De Sarkar, B. Johansson, and R. Ahuja, Appl. Phys. Lett. 105, 123903 (2014).

${ }^{178}$ B. Dunn, H. Kamath, and J.-M. Tarascon, Science 334, 928 (2011).

${ }^{179}$ E. Yoo, J. Kim, E. Hosono, H.-S. Zhou, T. Kudo, and I. Honma, Nano Lett. 8, 2277 (2008).

${ }^{180}$ D. Das, S. Kim, K.-R. Lee, and A. K. Singh, Phys. Chem. Chem. Phys. 15, $15128(2013)$

${ }^{181}$ G. A. Tritsaris, E. Kaxiras, S. Meng, and E. Wang, Nano Lett. 13, 2258 (2013).

${ }^{182}$ X. Tan, C. R. Cabrera, and Z. Chen, J. Phys. Chem. C 118, 25836 (2014).

${ }^{183}$ C. J. Tabert and E. J. Nicol, Phys. Rev. B 88, 085434 (2013).

${ }^{184} \mathrm{P}$. Gori et al., Energy Procedia 45, 512 (2014). 